\title{
TGLI1 transcription factor mediates breast cancer brain metastasis via activating metastasis-initiating cancer stem cells and astrocytes in the tumor microenvironment
}

\author{
Sherona R. Sirkisoon ${ }^{1}$, Richard L. Carpenter ${ }^{1,7}$, Tadas Rimkus ${ }^{1}$, Daniel Doheny ${ }^{1}$, Dongqin \\ Zhu $^{1}$, Noah R. Aguayo ${ }^{1}$, Fei Xing ${ }^{1,5}$, Michael Chan ${ }^{2,5}$, Jimmy Ruiz ${ }^{3,5}$, Linda J. Metheny- \\ Barlow $^{2,5}$, Roy Strowd ${ }^{4,5}$, Jiayuh Lin ${ }^{6}$, Boris Pasche ${ }^{1,5}$, Waldemar Debinski ${ }^{1,5}$, Kounosuke \\ Watabe $^{1,5}$, Hui-Wen Lo ${ }^{1,5,{ }^{*}}$ \\ ${ }^{1}$ Department of Cancer Biology, Wake Forest University School of Medicine, Winston-Salem, NC \\ ${ }^{2}$ Department of Radiation Oncology, Wake Forest University School of Medicine, Winston-Salem, \\ NC \\ ${ }^{3}$ Department of Hematology and Oncology, Wake Forest University School of Medicine, Winston- \\ Salem, NC \\ ${ }^{4}$ Department of Neurology, Wake Forest University School of Medicine, Winston-Salem, NC \\ ${ }^{5}$ Comprehensive Cancer Center, Wake Forest University School of Medicine, Winston-Salem, NC \\ ${ }^{6}$ Department of Biochemistry and Molecular Biology, University of Maryland School of Medicine, \\ Baltimore, MD. \\ ${ }^{7}$ Current Address: Department of Biochemistry and Molecular Biology, Indiana University School \\ of Medicine, Bloomington, IN
}

\begin{abstract}
Mechanisms for breast cancer metastasis remain unclear. Whether truncated glioma-associated oncogene homolog 1 (TGLI1), a transcription factor known to promote angiogenesis, migration and invasion, plays any role in metastasis of any tumor type has never been investigated. In this study, results of two mouse models of breast cancer metastasis showed that ectopic expression of TGLI1, but not GLI1, promoted preferential metastasis to the brain. Conversely, selective TGLI1 knockdown using antisense oligonucleotides led to decreased breast cancer brain metastasis (BCBM) in vivo. Immunohistochemical staining showed that TGLI1, but not GLI1, was increased in lymph node metastases compared to matched primary tumors, and that TGLI1 was expressed at higher levels in BCBM specimens compared to primary tumors. TGLI1 activation is associated with a shortened time to develop BCBM and enriched in HER2-enriched and triple-negative breast cancers. Radioresistant BCBM cell lines and specimens expressed higher levels of TGLI1, but not GLI1, than radiosensitive counterparts. Since cancer stem cells (CSCs) are radioresistant and
\end{abstract}

Users may view, print, copy, and download text and data-mine the content in such documents, for the purposes of academic research, subject always to the full Conditions of use:http://www.nature.com/authors/editorial_policies/license.html\#terms

*Corresponding Author: Hui-Wen Lo, Ph.D. Department of Cancer Biology, Wake Forest University School of Medicine, WinstonSalem, NC 27157, USA.

Competing Interest: None. 
metastasis-initiating cells, we examined TGLI1 for its involvement in breast CSCs and found TGLI1 to transcriptionally activate stemness genes CD44, Nanog, Sox2, and OCT4 leading to CSC renewal, and TGLI1 outcompetes with GLI1 for binding to target promoters. We next examined whether astrocyte-priming underlies TGLI1-mediated brain tropism and found that TGLI1-positive CSCs strongly activated and interacted with astrocytes in vitro and in vivo. These findings demonstrate, for the first time, that TGLI1 mediates breast cancer metastasis to the brain, in part, through promoting metastasis-initiating CSCs and activating astrocytes in BCBM microenvironment.

\section{Keywords}

TGLI1; breast cancer; cancer stem cells; brain metastasis; astrocytes

\section{INTRODUCTION}

Breast cancer is the second leading cause of cancer-related mortality in women and metastasis to distant organs result in $90 \%$ of breast cancer deaths ${ }^{1}$. Metastatic breast cancers occur in $20-30 \%$ of cases with a 5 -year survival rate of $22 \%^{2}$. The most common sites of breast cancer metastases are bone, lung, brain, and liver. HER2-enriched and triple-negative breast cancer (TNBC) subtypes have the highest propensity to metastasize to the brain, ranging from 20-30\% in HER2 and 45-60\% in TNBC ${ }^{2}$. Breast cancer brain metastasis (BCBM) constitutes $\sim 10-30 \%$ of metastatic breast cancer cases ${ }^{3}$. Current therapeutic options for women with BCBM include whole brain radiotherapy, surgical resection, and stereotactic radiosurgery ${ }^{4}$. Despite our current therapies and understanding of BCBM, breast cancer patients with brain metastases have a dismal prognosis of only 6-18 months of survival following diagnosis ${ }^{3}$. Mechanisms underlying BCBM remain poorly understood.

Our laboratory discovered a truncated form of glioma-associated oncogene homolog 1 (TGLI1 or tGLI1) and we have demonstrated that it is a gain-of-function GLI1 zinc-finger transcription factor ${ }^{5-10}$. TGLI1 is an alternatively spliced variant of GLI1, as a result of a 41 amino acid in-frame deletion, which retains all of the functional domains as GLI1, and is activated by the Shh-PTCH1-SMO signaling axis ${ }^{8}$. We have shown that TGLI1 is expressed in GBM and breast cancer, but is not expressed in normal brain or breast tissue ${ }^{5,8,9,11}$. Consistent with our findings, TGLI1 was reported to be expressed in highly invasive hepatocellular carcinoma but is not detected in normal hepatocytes ${ }^{12}$. TGLI1 has the ability to regulate GLI1 target genes but gains the ability to upregulate seven target genes which are not regulated by GLI1 ${ }^{5-10}$, namely, VEGF-A, VEGF-C, VEGFR2, TEM7, HPSE, CD24, and CD44 resulting in increased growth, angiogenesis, migration and invasion of glioma cells in vivo and breast cancer cells in vitro. In line with our results, VEGFR2 was reported to be transcriptionally activated by TGLI1 in TNBC cells leading to increased angiogenesis ${ }^{13}$. Whether TGLI1 plays any role in metastasis for any tumor type has never been investigated.

In this study, we aimed to determine whether TGLI1 plays a role in breast cancer metastasis. Through the use of three in vivo mouse models of metastasis, we observed that TGLI1 
promotes BCBM and facilitates the intracranial growth of breast cancer cells. We further observed that TGLI1 activation correlates with shortened metastasis-free survival and that TGLI1 expression is enriched in BCBM patient samples. Moreover, we found TGLI1 to play an important role in radioresistance and cancer stem cells (CSCs), through upregulating stemness gene expression and that TGLI1 outcompetes GLI1 for binding to target gene promoters. In agreement with an important role for astrocytes in intracranial tumor growth, TGLI1-positive CSCs strongly activate and interact with astrocytes and TGLI1-positive BCBM tumors strongly activate astrocytes in mouse brain. Lastly, knockdown of TGLI1 led to decreased cancer stemness in vitro and reduced BCBM in vivo. Collectively, our study defined TGLI1 as a novel mediator of BCBM, breast CSCs, activation of astrocytes in $\mathrm{BCBM}$ microenvironment, and resistance to radiation therapy.

\section{MATERIALS AND METHODS}

\section{Cell lines, tumor specimens, and expression plasmids}

Human breast cancer cell lines MCF10A, MCF7, and BT20 were purchased from ATCC (Manassas, VA) and cultured as specified by ATCC. HMLE, MDA-MB-231, and brain metastatic MDA-MB-231-BRM cell lines are from the Weinberg and Massagué laboratories, respectively. The brain metastatic cell line (SKBRM), radio-resistant cell lines (SKBRM-RR and 231BRM-RR), and E6/E7/hTERT immortalized human astrocytes were kind gifts from Drs. Fei Xing and Kounosuke Watabe. BC tissue microarrays (BR10010, BR952, BC01116) were purchased from US Biomax (Rockville, MD). BCBM patient specimens are from the Wake Forest Tumor Tissue Core and Dr. Kounosuke Watabe. The expression plasmids for GLI1 and TGLI1 were previously developed in our laboratory ${ }^{8}$.

\section{Animal studies}

All animal experiments were conducted as approved by the Wake Forest Institutional Animal Care and Use Committee. Isogenic MDA231 lines with vector, GLI1, or TGLI1, and SKBRM with negative control oligos or TGLI1-AS-ON $\left(1 \times 10^{5}\right.$ cells $)$ were injected into the left cardiac ventricle of female $\mathrm{nu} / \mathrm{hu}$ mice and monitored bi-weekly using an In Vivo Imaging System (IVIS). Intracranial implantations were performed as we described previously ${ }^{9}$. Briefly, female $\mathrm{nu} / \mathrm{nu}$ mice were anesthetized with ketamine/xylazine and isogenic cells $\left(2 \times 10^{4}\right.$ cells $/ 5 \mathrm{uL}$ PBS $)$ were stereotactically implanted into the right frontal lobe of the brain. Mice were monitored weekly using IVIS.

\section{Selective knockdowns of GLI1 and TGLI1}

Control or TGLI1 specific locked nucleic acid (LNA) antisense oligonucleotides (AS-ON) were custom designed and purchased from Qiagen. Knockdown of TGLI1 was conducted as described previously ${ }^{9}$. Briefly, BT20 or SKBRM cells were transfected for 48 hours with control or TGLI1 AS-ON using Lipofectamine 2000 (Invitrogen) and then cells were harvested for qRT-PCR, seeded for mammosphere assay, or intracardially injected into female $n u / n u$ mice described above. 


\section{Statistical analyses}

Results are represented as \pm SE. One-way ANOVA, Fisher's Exact Test, Log-Rank test, and student's $t$-test were performed using GraphPad Prism 5.

\section{RESULTS}

\section{TGLI1 promotes brain metastasis of TNBC in an intracardiac injection metastasis mouse model}

A role of TGLI1 in metastasis in any tumor type has not been investigated. To determine the effects of TGLI1 on metastasis, we generated isogenic TNBC MDA-MB-231 cell lines to overexpress TGLI1, GLI1 or control vector using lentiviral constructs ${ }^{9}$ and the firefly luciferase reporter. Stable expression of TGLI1 or GLI1 was confirmed using western blotting (Fig. 1A). Exogenous GLI1 and TGLI1 were expressed at physiologically relevant levels as indicated by comparable levels of GLI1 and TGLI1 in mammary fat pad (MFP) xenografts and primary breast carcinoma samples (Fig. 1B). Immunohistochemistry was conducted using TGLI1- and GLI1-specific antibodies we previously developed and validated $^{6,7,9,10}$. Of note, commercially available GLI1 antibodies detect both GLI1 and TGLI1 isoforms. Expression of TGLI1 in MDA-231-TGLI1 MFP tumor was equivalent to that detected in breast cancer PDXs as indicated by western blots (Fig. 1C); BCM-2174 (TNBC) and BCM-3963 (HER2-enriched) PDXs were shown to undergo brain metastasis ${ }^{14}$. We next used an experimental metastasis model to mimic several stages of metastasis including evasion of anoikis, intravasation, and colonization to distant organs, mainly to the brain and bone. For this, the isogenic lines were injected into the left ventricle of athymic female mice and the mice were imaged biweekly using an In Vivo Imaging System (IVIS). Representative bioluminescent images showed TGLI1 preferentially increased breast cancer metastasis to the brain compared to GLI1 and vector groups (Fig. 1D). To confirm bioluminescent signals were strictly from the brain or bone, but not surrounding tissues, we imaged the brain and bones ex vivo and we observed TGLI1 mice to have increased brain metastasis (Fig. 1E-G), but reduced bone metastasis compared to the vector group (Fig. 1FG). We further found the TGLI1 group to have the greater number of tumor foci among the three groups (Fig. 1H-I). These results suggest that TGLI1 promotes brain metastasis of circulating breast cancer cells.

\section{TGLI1 promotes the growth of TNBC in mouse brain in an intracranial injection mouse model}

We next evaluated the effects of TGLI1 on breast cancer intracranial growth using an intracranial implantation model. The isogenic MDA-MB-231 cell lines were implanted into the brain of female athymic mice and imaged weekly. The results showed TGLI1-expressing breast cancer cells were more tumorigenic than those with vector or GLI1 (Fig. 2A-B). TGLI1-positive brain metastases were significantly larger than GLI1 and vector tumors (Fig. 2C). Mouse brains carrying TGLI1-expressing BCBM were visibly larger and highly vascularized (Fig. 2D). We next examined tumors for expression of GLI1 and TGLI1 via immunohistochemistry (IHC), and confirmed their expression (Fig. 2E). TGLI1-expressing BCBM were more invasive as indicated by the hematoxylin and eosin (H\&E) staining, more vascularized (CD31 IHC; Fig. 2F), and more proliferative (Ki-67 IHC; Fig. 2G) compared to 
vector and GLI1 xenografts. These results indicate that TGLI1 promotes intracranial growth, invasion and angiogenesis of TNBC cells.

\section{Activation of TGLI1 is enriched in HER2-enriched and triple-negative breast cancers and associated with poor metastasis-free survival}

Whether TGLI1 target genes are associated with breast cancer patient outcomes remains unknown. DNA microarray probes do not distinguish TGLI1 from GLI1 transcripts. Therefore, we generated a TGLI1 activation signature (tGAS-6) containing six validated TGLI1 target genes (VEGFA, VEGFC, VEGFR2, TEM7, HPSE, and CD24) known to be regulated by TGLI1 but not GLI1 ${ }^{5-8,14}$ and determined whether tGAS-6 is correlated with patient survival using two large datasets retrieved from The Cancer Genome Atlas (TCGA) and Gene Expression Omnibus (GEO). The cDNA microarray probes used for GLI1 expression in both platforms detect both GLI1 and TGLI1 together. We found that TGLI1 is activated to a higher extent in HER2 and TNBC subtypes compared to luminal A/B subtypes in both TCGA and GEO datasets, whereas GLI1/TGLI1 expression was decreased in both datasets (Fig. 3A-B). Kaplan-Meier analysis of the GEO dataset indicated that patients with high tGAS-6, but not high GLI1/TGLI1 expression, had shortened overall metastasis-free survival (MFS; Fig. 3C), shortened brain MFS (Fig. 3D), and shortened lung and bone MFS (Supplementary Fig. 1A-B). These results indicated that TGLI1 activation is common in HER2-enriched breast cancer and TNBC, and associated with poor MFS, including brain MFS.

\section{Expression of TGLI1 is increased in lymph node metastases and BCBM samples compared to primary breast carcinomas}

A systematic analysis for TGLI1 expression, in comparison to GLI1, of breast cancer specimens has not been carried out. Analysis of primary breast carcinomas for TGLI1 and GLI1 protein expression by IHC indicated that TGLI1 expression was significantly higher in lymph node-positive, $\mathrm{LN}(+)$, primary breast tumors compared to $\mathrm{LN}(-)$ tumors, and that in LN(+) tumors, TGLI1 levels were higher than GLI1 expression (Fig. 3E). Furthermore, we immunostained 47 pairs of primary breast carcinomas and their matched LN metastases and found that TGLI1 expression was higher in LN metastases than in matched primary tumors and that TGLI1 levels were higher than GLI1 expression in both primary tumors and LN metastases (Fig. 3F-G). As shown in Supplementary Fig. 1C, GLI1 expression is higher in the paired lymph node metastases compared to the primary tumor but this trend was seen in $66 \%$ of the matched pairs and approximately $32 \%$ of the matched pairs have decreased GLI 1 expression. TGLI1 expression was higher than GLI1 in $\mathrm{LN}(+)$ breast cancer in a pairwise analysis (N=57; Fig. 3H). Next, we analyzed 37 BCBM specimens via IHC and compared their TGLI1/GLI1 expression levels to those in primary breast tumors; the results showed that TGLI1 levels were higher in BCBM than primary tumors (Fig. 3I). Pairwise analysis showed that in BCBM, TGLI1 was expressed at higher levels than GLI1 (Fig. 3J-K). These results showed that TGLI1 expression is increased in LN metastases and BCBM samples compared to primary breast carcinomas, suggesting its important role in BCBM. 


\section{TGLI1 contributes to BCBM radioresistance}

Standard treatment modalities for patients with BCBM include surgical resection, whole brain radiotherapy, and stereotactic radiosurgery ${ }^{4}$. Whether TGLI1 has any effect on radioresistance for any tumor type has never been investigated. Here, we evaluated the expression levels of TGLI1 and GLI1 in HER2-enriched brain-trophic (SKBRM) cells treated with 0, 2, or 5 Gray (Gy) of radiation. As shown in Fig. 4A, TGLI1 expression was significantly induced by radiation in a dose-dependent fashion whereas GLI1 expression was not significantly altered. We further observed that TGLI1 was expressed at higher levels in radioresistant SKBRM (SKBRM-RR) and MDA-MB-231-BrM (231BRM-RR) cells than the radiosensitive parental lines (Fig. 4B), whereas GLI1 levels were reduced in the radioresistant lines (Fig. 4C). Since radioresistance is an intrinsic hallmark of CSCs and the Hh pathway is an important mediator of tumorigenesis and stemness ${ }^{15}$, we determined whether TGLI1 contributes to the radioresistant phenotype of breast CSCs. We grew SKBRM-RR and SKBRM cells as mammospheres and evaluated levels of TGLI1; results showed that TGLI1 was expressed at higher levels in SKBRM-RR mammospheres than SKBRM mammospheres, whereas GLI1 expression was significantly decreased (Fig. 4D). Further shown in Fig. 4E-F, we observed that TGLI1 overexpression rendered radiosensitive stem cells (mammosphere) resistant to irradiation, and radioresistant stem cells were more resistant to irradiation. Furthermore, we confirmed these findings using 20 radiosensitive and 16 radioresistant BCBM samples by IHC. Radiosensitive samples were from patients that did not have recurrence one year after gamma-knife radiosurgery whereas radioresistant BCBMs were from patients with recurrence within one year of gamma-knife treatment. As shown in Fig. 4G-I, TGLI1 expression was significantly higher in radioresistant BCBMs than in radiosensitive samples whereas GLI1 was not differentially expressed between the two groups. Collectively, these data suggest a novel role that TGLI1 plays in radioresistance of BCBM.

\section{TGLI1 promotes breast CSCs; TGLI1-positive stem cells activate human astrocytes}

To elucidate the mechanism by which TGLI1 mediates BCBM and radioresistance, we examined the effects of TGLI1 on breast CSCs as they are resistant to radiation and are considered the metastasis-initiating cells ${ }^{16}$. Here, we determined TGLI1 expression levels in monolayer versus mammosphere (stem cell population) culture, and found TGLI1 to be induced by mammosphere culture in three different breast cancer cell lines, including luminal A (MCF-7), TNBC basal (BT-20), and brain-trophic HER2-enriched (SKBRM; Fig. 5A-B). As shown in Supplementary Fig. 2A, TGLI1 expression in monolayer was not induced by mammosphere media, indicating TGLI1 induction was due to CSCs and not growth factors present in the media. Furthermore, TGLI1 overexpression in MDA-MB-231 cells led to increased stem cell population defined by CD44high/CD24low (Fig. 5C). Ectopic expression of TGLI1 enhanced the mammosphere-forming ability of BT-20 cells (Fig. 5DF), SKBRM cells (Fig. 5G-I), and promoted mammosphere-forming ability of two immortalized normal mammary epithelial cell lines, MCF10A and HMLE (Supplementary Fig. 2B-C), and enhanced the anchorage-independent growth of HMLE cells (Supplementary Fig. 2D). 
Reactive astrocytes have been shown to support the arrest, extravasation, and growth of breast cancer cells in the brain ${ }^{17}$. To explore whether TGLI1-expressing breast CSCs have any effect on astrocyte activation, we stimulated human astrocytes with conditioned medium from SKBRM cells grown in monolayer or mammosphere culture. As shown in Fig. 5J-K, TGLI1-positive mammospheres significantly activated astrocytes as indicated by increased glial fibrillary acidic protein (GFAP) expression. Further shown in Fig. 5L, astrocytes only survived and interacted with TGLI1-positive breast CSCs under the sphere-forming stem cell culturing condition. Immunostaining of mouse BCBM tumors (described in Fig. 2) revealed TGLI1-positive tumors significantly activated astrocytes in vivo (Fig. 5M-N). In contrast, no difference was observed between vector and GLI1 groups. Together, these results indicated that TGLI1 promotes breast CSCs and that TGLI1-positive stem cells and tumors can activate astrocytes in vitro and in vivo respectively, a mechanism underlying TGLI1-mediated brain metastasis.

\section{TGLI1 upregulates stemness genes in breast cancer cells}

Whether TGLI1 transcriptionally regulates stemness genes in breast cancer has never been investigated. Here, we determined the extent to which TGLI1 mediates breast CSCs by examining the ability of TGLI1 to regulate expression of four genes that are important for pluripotency and stem cell self-renewal, namely, Nanog, SOX2, OCT4, and CD44 ${ }^{18,} 19$. GLI1 has been previously shown to directly regulate expression of SOX2 and Nanog 20,21 . We recently reported that TGLI1, but not GLI1, transcriptionally upregulated CD44 gene expression in glioma stem cells ${ }^{9}$. Whether OCT 4 can be regulated by TGLI1 has not been reported. To identify whether these genes are associated with BCBM, we used Kaplan-Meier analysis and the same GEO datasets used in Fig. 3C-D, and found that SOX2 and OCT4, but not Nanog and CD44, were associated with shortened brain MFS (Supplementary Fig. 3A). We found that TGLI1 upregulates expression of all four stemness genes (Fig. 6A-C \& Supplementary Fig. 3B-D). Of note, Nanog, SOX2, and OCT4 were significantly upregulated by TGLI1 in comparison to both vector and GLI1 in three cell lines. Consistent with the mRNA results, protein levels of all four stemness genes were enhanced by TGLI1 in SKBRM cells (Fig. 6D). We next examined whether TGLI1 regulates expression of GLI2 target genes ${ }^{22-24}$ and found TGLI1 to upregulate GLI2 target genes to a higher extent than GLI1 (Supplementary Fig. 3E). Promoter luciferase reporter assay showed that TGLI1 possessed the ability to transactivate the promoters of Nanog and SOX2 (two known GLI1 target genes; Fig. 6E-F). Using chromatin immunoprecipitation (ChIP) and promoter luciferase reporter assays, we further found that TGLI1 significantly bound to and transactivated CD44 (Fig. 6G-H) and OCT4 (Fig. 6I-J) promoters. Interestingly, we found that TGLI1 bound to the OCT4 promoter to a similar degree as GLI1; however, TGLI1 was able to transactivate the OCT4 promoter more than GLI1 to increase OCT4 mRNA and protein expression. Although GLI family of proteins do not dimerize with each other, we determined whether TGLI1 and GLI1 compete to bind to target gene promoters. We used isogenic MDA-MB-231 cells overexpressing both GLI1 and TGLI1, and performed ChIP assay using TGLI1- and GLI1-specific antibodies. As shown in Fig. 6K, TGLI1 preferentially binds to the OCT4 promoter in the presence of GLI1, indicating that TGLI1 outcompetes GLI1 for binding to target gene promoters. Collectively, these results demonstrate that TGLI1 is a gain-of-function GLI1 transcription factor with the ability to 
upregulate stemness genes and that TGLI1 is a novel transcriptional activator of Nanog, SOX2, and OCT4.

\section{Eight-gene TGLI1 activation signature (tGAS-8) is predictive of breast cancer metastasis and radioresistance}

We recently identified two new TGLI1 target genes, CD44 and OCT4, which prompted us to create the 8-gene TGLI1 activation signature (tGAS-8) and determine its utility as a prognostic marker for breast cancer metastasis. Using tGAS-8 and the GEO datasets used earlier in Fig. 3, we found that tGAS-8 was more enriched in the HER2 and TNBC subtypes than the luminal A/B subtypes (Supplementary Fig. 3F). Metastatic breast tumors had higher tGAS-8 than non-metastatic counterparts (Fig. 6L \& Supplementary Fig. 3G). Patients with brain or lung metastases had higher tGAS-8 in their primary tumors compared to those with bone metastases and no metastases (Supplementary Fig. 3H). In contrast, GLI1/TGLI1 mRNA was decreased in breast cancer with metastases (Supplementary Fig. 3G-H). Furthermore, Kaplan-Meier analyses showed that patients with high tGAS-8 have a shortened overall MFS, as well as, shortened brain, lung, and bone MFS, compared to those with low tGAS-8 (Fig. 6M \& Supplementary Fig. 3I). Next, we investigated whether tGAS- 8 is associated with metastasis and radioresistance using Gene Set Enrichment Analysis (GSEA) and the same GEO datasets. For this, we retrieved previously established signatures for cancer metastasis ${ }^{25}, \mathrm{BCBM}^{26,27}, \mathrm{EMT}^{28}$, breast cancer stemness ${ }^{29}$, and breast cancer radioresistance ${ }^{30}$ (Fig. $6 \mathrm{~N} \&$ Supplemental Fig. 3J-M). The results showed that breast tumors with high tGAS-8, but not GLI1/TGLI1 mRNA, were enriched for all five signatures. Taken together, these results indicated that tGAS- 8 can be used as a prognostic indicator for metastatic breast cancer and radioresistance.

\section{TGLI1 Knockdown suppresses breast CSCs in vitro and reduces BCBM in vivo}

To further validate the effects of TGLI1 on breast CSCs, we used validated antisense oligonucleotides (AS-ON) to selectively knockdown TGLI1 in BT20 and SKRBM cells then determined the effect on mammosphere-forming ability. Consistent with our recent report ${ }^{9}$ the AS-ON led to selective knockdown of TGLI1 in both cell lines and significantly decreased the number of mammospheres compared to control (Fig. 7A-B). TGLI1 knockdown decreased expression of the recently identified TGLI1 target genes, OCT4 and CD44 (Fig. 7C). To determine the functional role of TGLI1 on breast cancer metastasis, we knocked down TGLI1 in brain trophic SKBRM cells and performed intracardiac injections to study brain and bone metastasis (Fig. 7D). Ex vivo analysis of the brain and bone revealed a significant decrease in brain metastases in the TGLI1 knockdown group (Fig. 7E-F) but no difference in bone metastases (Fig. 7E-G). These results further support the novel role of TGLI1 in mediating breast cancer stem cells and BCBM.

\section{DISCUSSION}

In this study we made the following novel and impactful observations: (1) TGLI1 promotes breast cancer metastasis to brain, and intracranial growth of breast cancer cells in two mouse models of breast cancer metastasis; (2) TGLI1 activation is increased in HER2-enriched and TNBC subtypes and is associated with shortened brain MFS; (3) TGLI1 expression is 
enriched in LN metastases and BCBM specimens compared to primary breast carcinomas; (4) TGLI1 is increased in radioresistant BCBM cell lines and specimens compared to radiosensitive counterparts, and contributes to resistance of BCBM cells; (5) TGLI1 promotes mammosphere formation in breast cancer cells and normal mammary epithelial cells; (6) TGLI1 mediates breast CSCs by transcriptionally activating cancer stemness gene expression; (7) TGLI1 upregulates expression of GLI2 target genes; (8) TGLI1 outcompetes with GLI1 to bind to OCT4 gene promoter; (9) TGLI1-postive breast CSCs strongly activate and interact with astrocytes in vitro and in vivo; and (10) TGLI1 knockdown decreases mammosphere formation, decreases stemness gene expression, and reduces breast cancer metastasis to the brain. Through making these significant observations, our study defines TGLI1 as a novel mediator of brain metastasis, astrocyte activation, and resistance to radiation therapy in breast cancer, thereby providing novel insights into the molecular mechanisms for BCBM.

We observed in this study that TGLI1 promotes breast CSCs. This is consistent with previous studies reporting that a subpopulation of breast CSCs can be characterized by cellsurface expression of CD $44^{\text {high}} / \mathrm{CD} 24^{\text {low }} 31,32$. Interestingly, we have previously reported that both CD44 and CD24 can be transcriptionally activated by TGLI $1{ }^{5}$ while this study shows TGLI1 to increase the CD44 high/CD2 $4^{\text {low }}$ stem cell subpopulation. We speculate that TGLI1 may have increased the expression of nuclear CD24 but not cell-surface CD24 expression. It has been recently discovered that biologically active CD24 is localized in the nucleus of cancer cells with low cell-surface CD24 expression. Importantly, they found that nuclear CD24 correlated with metastasis and that a nuclear CD24 gene signature was predictive of shortened survival for breast cancer patients ${ }^{33}$. In agreement with these findings, and the role of the TGLI1-CD24 signaling axis in promoting cancer migration and invasion ${ }^{5,8}$ and metastasis (Figs. 1-2), other groups have shown that CD24-positive breast cancers are associated with tumor progression and poor survival ${ }^{34,35}$. These results suggest that TGLI1 may increase nuclear CD24 expression but reduce cell-surface CD24 expression, and thereby contributes to breast CSCs and cancer metastasis. This possibility should be addressed in future studies.

Our results from three mouse models of breast cancer metastasis indicate that TGLI1 directs breast cancer metastasis to the brain and promotes breast cancer growth once in the brain. We also demonstrate TGLI1-positive CSCs strongly activate and interact with astrocytes in vitro and TGLI1-positive BCBM tumors activate astrocytes in vivo. The ability of TGLI1 to activate astrocytes should be investigated further. Apart from mediating breast CSCs to drive $\mathrm{BCBM}$ and activating astrocytes to alter the tumor microenvironment, another potential mechanism by which TGLI1 may mediate BCBM is through regulating exosomal microRNAs. Through secretion of exosomes, small extracellular vesicles containing growth factors, DNA, and microRNAs, primary tumors can prime distant organs for colonization of tumor cells ${ }^{36}$. Whether TGLI1-positive tumors secrete exosomal microRNAs to prime the brain for colonization remains to be investigated. Furthermore, results from our bioinformatic analysis implicate TGLI1 in facilitating lung and bone metastases (Supplementary Figs. 1 and 3). Using one cell line in our in vivo model, to overexpress TGLI1, we did not observe significant increase in lung (not shown) or bone metastases between vector, GLI1 or TGLI1 groups suggesting that TGLI1-positive breast cancers 
preferentially metastasize to the brain. Similarly, TGLI1 knockdown in a brain trophic HER2-enriched cell line (SKBRM) did not lead to significant differences in lung and bone metastases. Future experiments using additional breast cancer cell lines, or genetically engineered mouse models, are needed to further define the impact of TGLI1 on breast cancer metastasis in the context of organ preference.

We found that TGLI1 overexpression transformed normal mammary epithelial cells resulting in increased mammosphere formation and anchorage-independent growth, key features of CSCs. This finding suggests TGLI1 may play an oncogenic role for mammary tumorigenesis. Future studies examining the role of TGLI1 in malignant transformation of normal mammary cells are needed. To meet this need, conditional transgenic mouse models may be established to allow for GLI1 gene knocked out and human TGLI1 gene knocked in. These floxed mice can then be crossed with mammary-specific Cre mice such as MMTV-cre mice. This transgenic mouse model will shed new light on the effects of TGLI1 on malignant transformation of mammary cells, breast tumor growth, and progression.

We identified OCT4 as a novel transcriptional target of TGLI1 in breast cancer cells (Fig. 6). OCT4 is an established stemness gene ${ }^{18}$. This novel TGLI1-OCT4 signaling axis represents a previously undescribed molecular mechanism underlying breast CSCs. Aberrant expression of OCT4 has been shown to promote breast cancer progression, chemoresistance, and metastasis, and to be associated with $\mathrm{TNBCs}^{37}$. In contrast, OCT4 overexpression resulted in decreased migration and invasion in vitro, as well as, decreased lung metastases in vivo ${ }^{38}$. Interestingly, a study demonstrated that OCT4 is expressed in adult stem cells and has been shown to mediate adult neural stem cell differentiation into dopaminergic neurons ${ }^{39}$. The important role of OCT4 in the adult brain may contribute to the observed preferential brain metastasis of TGLI1-positive breast cancers. Future studies are needed to address this possibility.

To fully understand the mechanism by which TGLI1 promotes breast CSCs, RNA-seq will be carried out in future to identify additional TGLI1-specific stemness genes. The current study identified OCT4 as a stemness gene that underlies TGLI1-mediated breast cancer stemness. This study also confirmed the existence of the TGLI1-CD44 signaling axis in breast cancer which was first discovered in GBM stem cells ${ }^{9}$. Based on these findings, TGLI1 may mediate breast CSCs through upregulating both OCT4 and CD44 plus additional genes. We demonstrate that TGLI1 outcompetes GLI1 for binding to the OCT4 gene promoter which may contribute to the gain-of-function ability of TGLI1. We further uncovered that TGLI1, but not GLI1, upregulates GLI2 target genes including PDGFR $\beta$, FGF19, and ARHGEF16. PDGFR $\beta$ is an important neuroprotective factor during brain injury and is associated with breast cancer brain metastasis and gastric cancer stem cells $^{24,40}$. FGF19, the human ortholog of FGF15, is essential for embryonic brain development ${ }^{41}$ and overexpression of FGF19 is implicated in breast cancer progression and metastasis ${ }^{42}$. ARHGEF16 was recently shown to promote glioma cell migration and proliferation ${ }^{22}$. Upregulation of these GLI2 target genes by TGLI1 further contributes to the aggressiveness of TGLI1. Future studies are needed to address how TGLI1 regulates GLI2 target genes. One possibility for TGLI1's gain-of-function ability is that TGLI1 may interact with other transcription factors to co-regulate gene expression. Another possibility is that 
TGLI1 may bind to different consensus sites that GLI1 cannot bind. However, these possibilities will need to be thoroughly investigated in future studies.

In summary, our current study reports important findings on the novel functions of TGLI1 and mechanisms contributing to BCBM, stem cells, reprogramming of tumor microenvironment via astrocyte activation, and radioresistance. The results from this study, together with previous studies, have further strengthened the rationale to further investigate the roles of TGLI1 in regulating exosomal microRNAs in the context of BCBM and mammary tumorigenesis, as well as, identify the mechanism that underlie the gain-offunction transcriptional ability of TGLI1.

\section{Supplementary Material}

Refer to Web version on PubMed Central for supplementary material.

\section{ACKNOWLEDGEMENTS}

We thank Dr. Shadi Qasem for providing pathology support. We acknowledge the funding support from NIH grants R01NS087169 (to H-WL), F31CA224805-01A1 (to SRS, H-WL), T32CA079448 (to SK, RLC), R01NS087169-3S1 (to H-WL, SRS), P30CA012197 (to BP; core facility), and DoD grants W81XWH-17-1-0044 (to H-WL) and W81XWH-19-1-0072 (to H-WL).

\section{REFERENCES}

1. Siegel RL, Miller KD, Jemal A. Cancer statistics, 2018. CA: a cancer journal for clinicians 2018; 68: 7-30. [PubMed: 29313949]

2. Wu Q, Li J, Zhu S, Wu J, Chen C, Liu Q et al. Breast cancer subtypes predict the preferential site of distant metastases: a SEER based study. Oncotarget 2017; 8: 27990-27996. [PubMed: 28427196]

3. Ekici K, Temelli O, Dikilitas M, Halil Dursun I, Bozdag Kaplan N, Kekilli E. Survival and prognostic factors in patients with brain metastasis: Single center experience. Journal of BUON : official journal of the Balkan Union of Oncology 2016; 21: 958-963.

4. Niikura N, Hayashi N, Masuda N, Takashima S, Nakamura R, Watanabe K et al. Treatment outcomes and prognostic factors for patients with brain metastases from breast cancer of each subtype: a multicenter retrospective analysis. Breast cancer research and treatment 2014; 147: 103112. [PubMed: 25106661]

5. Cao X, Geradts J, Dewhirst MW, Lo HW. Upregulation of VEGF-A and CD24 gene expression by the tGLI1 transcription factor contributes to the aggressive behavior of breast cancer cells. Oncogene 2012; 31: 104-115. [PubMed: 21666711]

6. Carpenter RL, Paw I, Zhu H, Sirkisoon S, Xing F, Watabe K et al. The gain-of-function GLI1 transcription factor TGLI1 enhances expression of VEGF-C and TEM7 to promote glioblastoma angiogenesis. Oncotarget 2015; 6: 22653-22665. [PubMed: 26093087]

7. Han W, Carpenter RL, Lo H-W. TGLI1 Upregulates Expression of VEGFR2 and VEGF-A, Leading to a Robust VEGF-VEGFR2 Autocrine Loop and Cancer Cell Growth. Cancer Hallmarks 2013; 1: $28-37$.

8. Lo HW, Zhu H, Cao X, Aldrich A, Ali-Osman F. A novel splice variant of GLI1 that promotes glioblastoma cell migration and invasion. Cancer research 2009; 69: 6790-6798. [PubMed: 19706761]

9. Rimkus TK, Carpenter RL, Sirkisoon S, Zhu D, Pasche BC, Chan MD et al. Truncated GliomaAssociated Oncogene Homolog 1 (tGLI1) Mediates Mesenchymal Glioblastoma via Transcriptional Activation of CD44. Cancer research 2018; 78: 2589-2600. [PubMed: 29463580]

10. Zhu H, Carpenter RL, Han W, Lo HW. The GLI1 splice variant TGLI1 promotes glioblastoma angiogenesis and growth. Cancer Lett 2014; 343: 51-61. [PubMed: 24045042] 
11. Sirkisoon SR, Carpenter RL, Rimkus T, Anderson A, Harrison A, Lange AM et al. Interaction between STAT3 and GLI1/tGLI1 oncogenic transcription factors promotes the aggressiveness of triple-negative breast cancers and HER2-enriched breast cancer. Oncogene 2018; 37: 2502-2514. [PubMed: 29449694]

12. Fan YH, Ding J, Nguyen S, Liu XJ, Xu G, Zhou HY et al. Aberrant hedgehog signaling is responsible for the highly invasive behavior of a subpopulation of hepatoma cells. Oncogene 2015; 35: 116. [PubMed: 25772244]

13. Di Mauro C, Rosa R, D’Amato V, Ciciola P, Servetto A, Marciano R et al. Hedgehog signalling pathway orchestrates angiogenesis in triple-negative breast cancers. British Journal Of Cancer 2017; 116: 1425. [PubMed: 28441382]

14. Zhang X, Claerhout S, Prat A, Dobrolecki LE, Petrovic I, Lai Q et al. A renewable tissue resource of phenotypically stable, biologically and ethnically diverse, patient-derived human breast cancer xenograft models. Cancer research 2013; 73: 4885-4897. [PubMed: 23737486]

15. Dahmane N, Sánchez P, Gitton Y, Palma V, Sun T, Beyna M et al. The Sonic Hedgehog-Gli pathway regulates dorsal brain growth and tumorigenesis. Development 2001; 128: 5201-5212. [PubMed: 11748155]

16. Wang T, Shigdar S, Gantier MP, Hou Y, Wang L, Li Y et al. Cancer stem cell targeted therapy: progress amid controversies, 2015.

17. Lorger M, Felding-Habermann B. Capturing changes in the brain microenvironment during initial steps of breast cancer brain metastasis. The American journal of pathology 2010; 176: 2958-2971. [PubMed: 20382702]

18. Wang Z, Oron E, Nelson B, Razis S, Ivanova N. Distinct Lineage Specification Roles for NANOG, OCT4, and SOX2 in Human Embryonic Stem Cells. Cell Stem Cell 2012; 10: 440-454. [PubMed: 22482508]

19. Wang L, Zuo X, Xie K, Wei D. The Role of CD44 and Cancer Stem Cells. Methods in molecular biology (Clifton, NJ) 2018; 1692: 31-42.

20. Bora-Singhal N, Perumal D, Nguyen J, Chellappan S. Gli1-Mediated Regulation of Sox2 Facilitates Self-Renewal of Stem-Like Cells and Confers Resistance to EGFR Inhibitors in NonSmall Cell Lung Cancer(). Neoplasia (New York, NY) 2015; 17: 538-551.

21. Li Q, Lex RK, Chung H, Giovanetti SM, Ji Z, Ji H et al. The Pluripotency Factor NANOG Binds to GLI Proteins and Represses Hedgehog-mediated Transcription. The Journal of biological chemistry 2016; 291: 7171-7182. [PubMed: 26797124]

22. Huang D, Wang Y, Xu L, Chen L, Cheng M, Shi W et al. GLI2 promotes cell proliferation and migration through transcriptional activation of ARHGEF16 in human glioma cells. Journal of experimental \& clinical cancer research : CR 2018; 37: 247. [PubMed: 30305138]

23. Komada M, Saitsu H, Shiota K, Ishibashi M. Expression of Fgf15 is regulated by both activator and repressor forms of Gli2 in vitro. Biochemical and biophysical research communications 2008; 369: 350-356. [PubMed: 18279667]

24. Wang JX, Zhou JF, Huang FK, Zhang L, He QL, Qian HY et al. GLI2 induces PDGFRB expression and modulates cancer stem cell properties of gastric cancer. European review for medical and pharmacological sciences 2017; 21: 3857-3865. [PubMed: 28975979]

25. Zucchi I, Mento E, Kuznetsov VA, Scotti M, Valsecchi V, Simionati B et al. Gene expression profiles of epithelial cells microscopically isolated from a breast-invasive ductal carcinoma and a nodal metastasis. Proceedings of the National Academy of Sciences of the United States of America 2004; 101: 18147-18152. [PubMed: 15608061]

26. Bos PD, Zhang XH, Nadal C, Shu W, Gomis RR, Nguyen DX et al. Genes that mediate breast cancer metastasis to the brain. Nature 2009; 459: 1005-1009. [PubMed: 19421193]

27. Smid M, Wang Y, Zhang Y, Sieuwerts AM, Yu J, Klijn JGM et al. Subtypes of Breast Cancer Show Preferential Site of Relapse. Cancer research 2008; 68: 3108-3114. [PubMed: 18451135]

28. Liberzon A, Birger C, Thorvaldsdóttir H, Ghandi M, Mesirov Jill P, Tamayo P. The Molecular Signatures Database Hallmark Gene Set Collection. Cell Systems 2015; 1: 417-425. [PubMed: 26771021]

Oncogene. Author manuscript; available in PMC 2020 February 28. 
29. Akrap N, Andersson D, Bom E, Gregersson P, Stahlberg A, Landberg G. Identification of Distinct Breast Cancer Stem Cell Populations Based on Single-Cell Analyses of Functionally Enriched Stem and Progenitor Pools. Stem cell reports 2016; 6: 121-136. [PubMed: 26771357]

30. He DX, Xia YD, Gu XT, Jin J, Ma X. A transcription/translation-based gene signature predicts resistance to chemotherapy in breast cancer. Journal of pharmaceutical and biomedical analysis 2015; 102: 500-508. [PubMed: 25459950]

31. Abraham BK, Fritz P, McClellan M, Hauptvogel P, Athelogou M, Brauch H. Prevalence of CD44+/ CD24-/low cells in breast cancer may not be associated with clinical outcome but may favor distant metastasis. Clinical cancer research : an official journal of the American Association for Cancer Research 2005; 11: 1154-1159. [PubMed: 15709183]

32. Sheridan C, Kishimoto H, Fuchs RK, Mehrotra S, Bhat-Nakshatri P, Turner CH et al. CD44+/ CD24- breast cancer cells exhibit enhanced invasive properties: an early step necessary for metastasis. Breast cancer research : BCR 2006; 8: R59. [PubMed: 17062128]

33. Duex JE, Owens C, Chauca-Diaz A, Dancik GM, Vanderlinden LA, Ghosh D et al. Nuclear CD24 Drives Tumor Growth and Is Predictive of Poor Patient Prognosis. Cancer research 2017; 77: 4858-4867. [PubMed: 28674079]

34. Ahmed MA, Aleskandarany MA, Rakha EA, Moustafa RZ, Benhasouna A, Nolan C et al. A $\mathrm{CD} 44(-) / \mathrm{CD} 24(+)$ phenotype is a poor prognostic marker in early invasive breast cancer. Breast cancer research and treatment 2012; 133: 979-995. [PubMed: 22119938]

35. Wang Z, Wang Q, Wang Q, Wang Y, Chen J. Prognostic significance of CD24 and CD44 in breast cancer: a meta-analysis. The International journal of biological markers 2017; 32: e75-e82. [PubMed: 27470135]

36. Almeida MI, Reis RM, Calin GA. MicroRNA history: Discovery, recent applications, and next frontiers. Mutation Research/Fundamental and Molecular Mechanisms of Mutagenesis 2011; 717: 1-8. [PubMed: 21458467]

37. Gwak JM, Kim M, Kim HJ, Jang MH, Park SY. Expression of embryonal stem cell transcription factors in breast cancer: Oct4 as an indicator for poor clinical outcome and tamoxifen resistance. Oncotarget 2017; 8: 36305-36318. [PubMed: 28422735]

38. Shen L, Qin K, Wang D, Zhang Y, Bai N, Yang S et al. Overexpression of Oct4 suppresses the metastatic potential of breast cancer cells via Rnd1 downregulation. Biochimica et Biophysica Acta (BBA) - Molecular Basis of Disease 2014; 1842: 2087-2095. [PubMed: 25068817]

39. Deleidi M, Cooper O, Hargus G, Levy A, Isacson O. Oct4-induced reprogramming is required for adult brain neural stem cell differentiation into midbrain dopaminergic neurons. PloS one 2011; 6: e19926. [PubMed: 21655272]

40. Gril B, Palmieri D, Qian Y, Anwar T, Liewehr DJ, Steinberg SM et al. Pazopanib inhibits the activation of PDGFRbeta-expressing astrocytes in the brain metastatic microenvironment of breast cancer cells. The American journal of pathology 2013; 182: 2368-2379. [PubMed: 23583652]

41. Miyake A, Nakayama Y, Konishi M, Itoh N. Fgf19 regulated by Hh signaling is required for zebrafish forebrain development. Developmental biology 2005; 288: 259-275. [PubMed: 16256099]

42. Zhao X, Xu F, Dominguez NP, Xiong Y, Xiong Z, Peng H et al. FGFR4 provides the conduit to facilitate FGF19 signaling in breast cancer progression. Molecular carcinogenesis 2018; 57: 16161625. [PubMed: 30074276] 

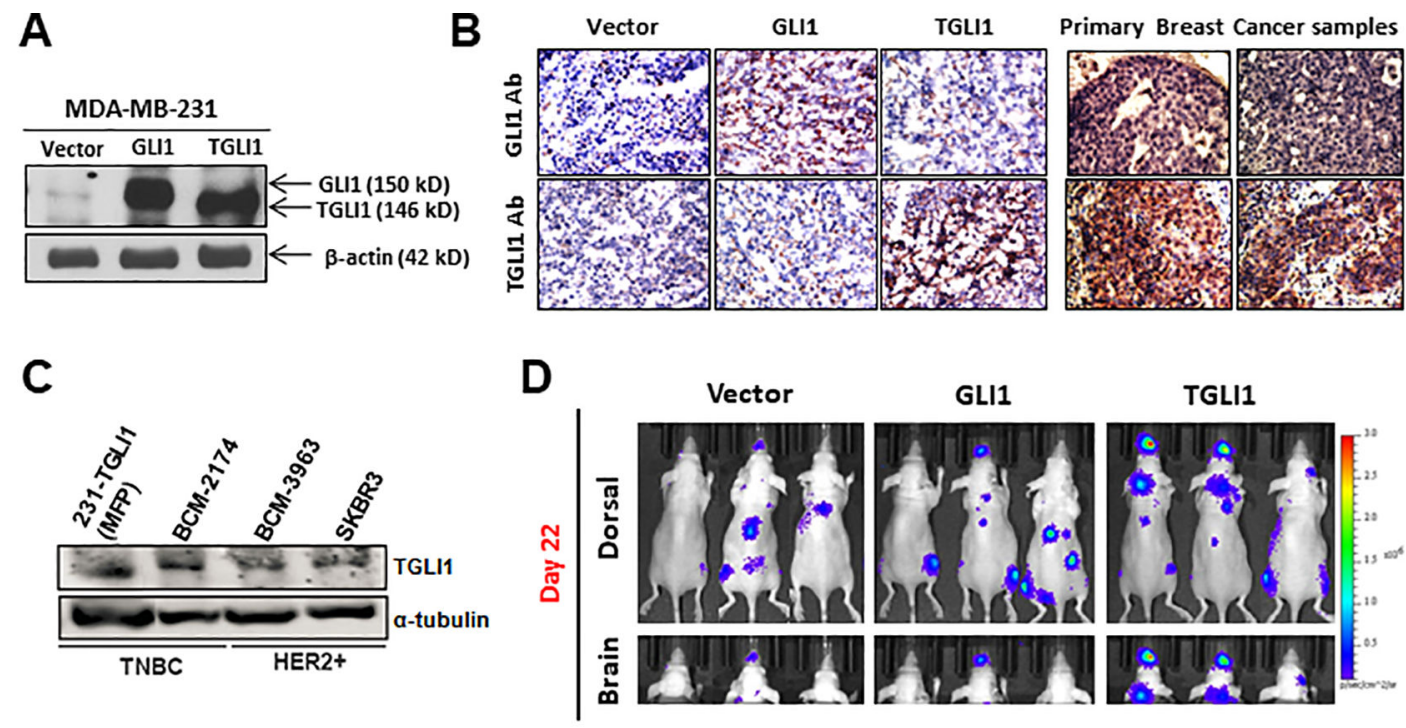

E

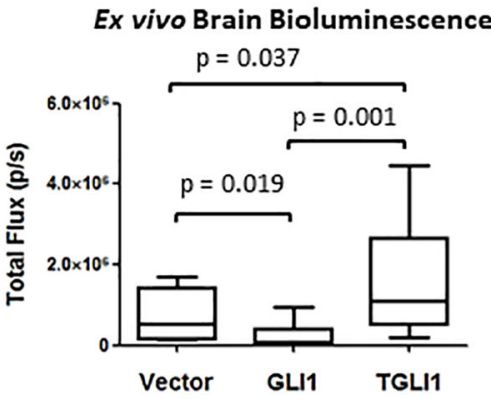

G

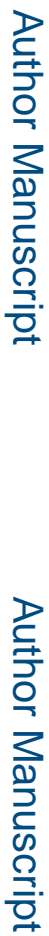
mouse model.
$\mathbf{F}$

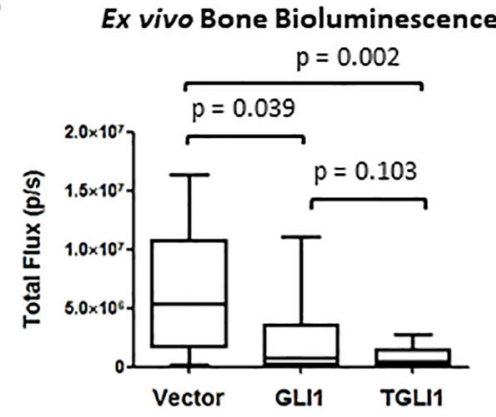

Figure 1: TGLI1 promotes brain metastasis of TNBC in an intracardiac injection metastasis

A) Western blot analysis of isogenic MDA-MB-231 cell lines. B) IHC analysis of mammary fat pad MDA-MB-231 xenografts from female athymic mice and primary breast cancer patient samples using GLI1- and TGLI1-selective antibodies. C) Expression of TGLI1 in MDA-MB-231-TGLI1 MFP xenograft was similar to that found in two breast cancer PDXs. Western blots using the TGLI1-specific antibody were conducted. D) Bioluminescent images of female athymic mice 22 days post intracardiac injection of isogenic MDA- 
MB-231 cell lines. Dorsal and brain views are shown. Twelve mice per group were used. Two mice in vector and one mouse in TGLI1 groups died during quantified. G) Tumor foci within mouse brains were determined via ex vivo brain imaging. H) Representative ex vivo IVIS images of brain and bone are shown. Student's $t$-test was used to compute p-values for panels $\mathrm{E}$ and $\mathrm{F}$. 
A

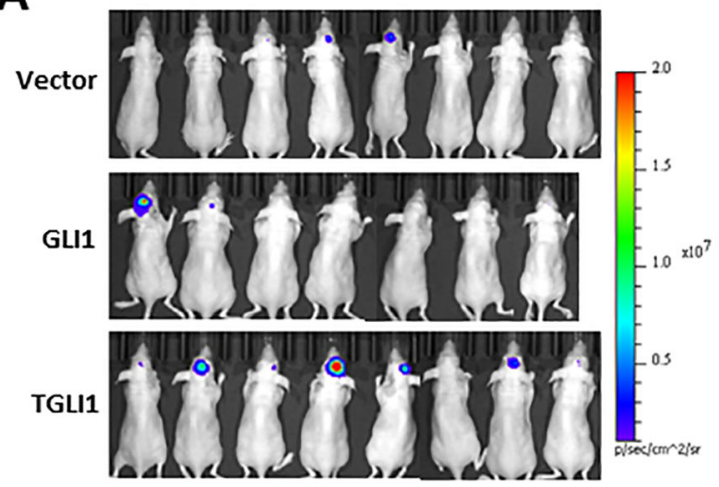

B

\begin{tabular}{|l|c|}
\hline \multicolumn{2}{|c|}{231 Intracranial Tumors } \\
\hline MDA-231-Vector & $2 / 8(25 \%)^{\star}$ \\
\hline MDA-231-GLI1 & $2 / 7(29 \%)^{+}$ \\
\hline MDA-231-TGLI1 & $7 / 8(88 \%)^{\star *+}$ \\
\hline
\end{tabular}

C Intracranial Bioluminescence

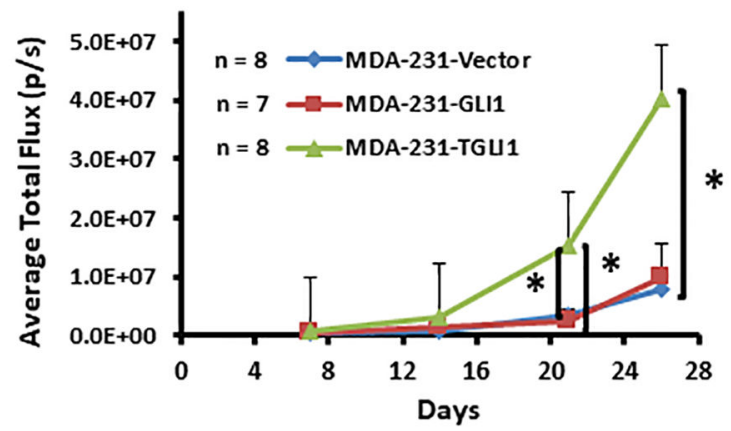

D

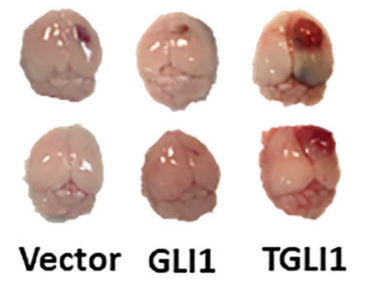

E
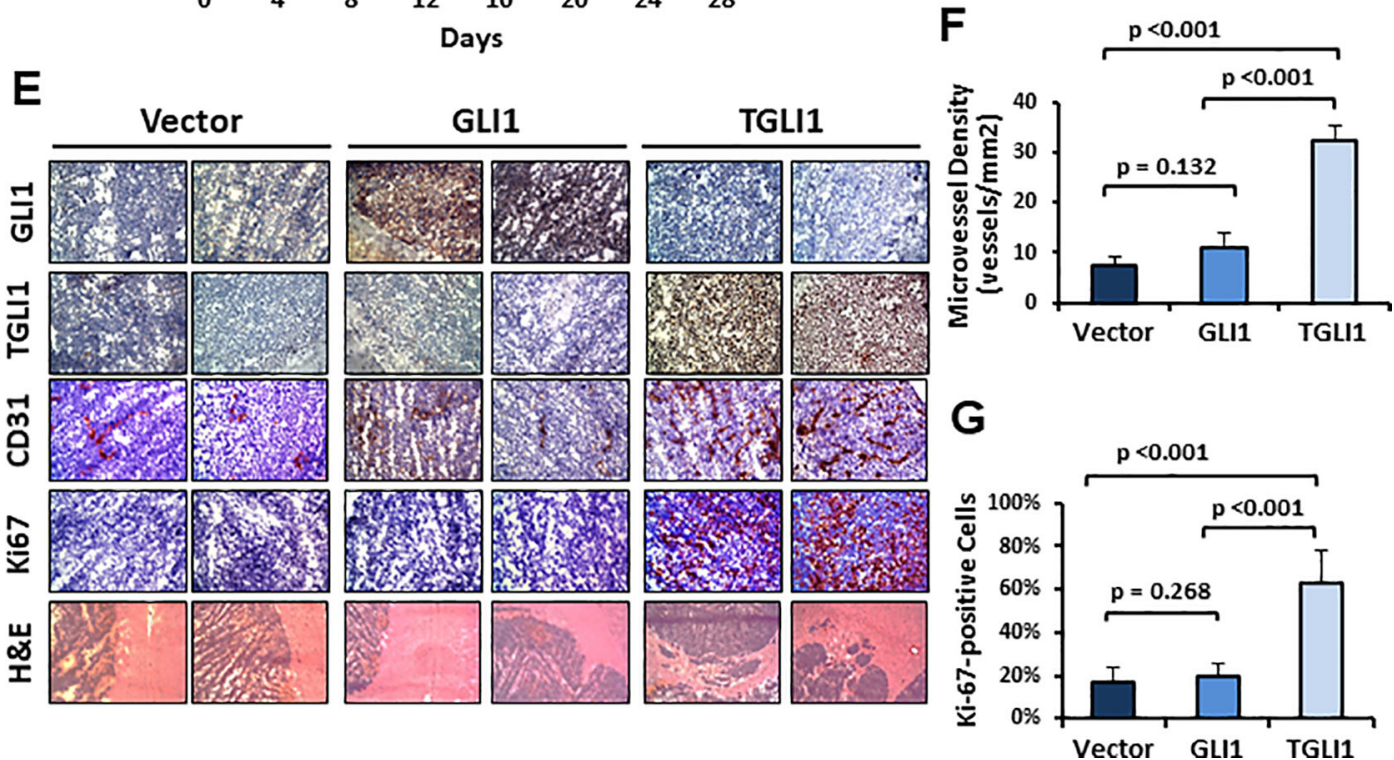

Figure 2: TGLI1 promotes TNBC growth in mouse brain in an intracranial injection mouse model.

Isogenic MDA-MB-231 lines were implanted into mouse brain and imaged weekly via an IVIS imager. Eight mice per group were used in which one mouse in the GLI1 group died of ketamine overdose. A) IVIS images of female athymic mice 3 weeks post intracranial implantation. B) Incidence of BCBM formation in each group. ${ }^{*},{ }^{+}$indicates $\mathrm{p}<0.05$ between vector and TGLI1 or GLI1 and TGLI1, respectively. Fisher's Exact Test was used. C) Growth curve for intracranial tumors. * indicates $\mathrm{p}<0.05$. D) Representative mouse 
brains showing TGLI1 BCBM being more vascularized. E) Analysis of BCBM for GLI1/ TGLI1 expression (IHC), invasiveness (H\&E), microvessel density (CD31 IHC) and proliferation index (Ki-67 IHC). F) Microvessel density. G) Proliferation Index. Student's $t$ test was used to compute $\mathrm{p}$-values for panels $\mathrm{F}$ and $\mathrm{G}$. 
A GEO

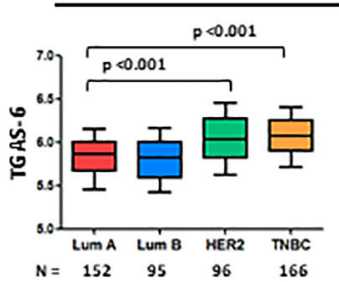

C
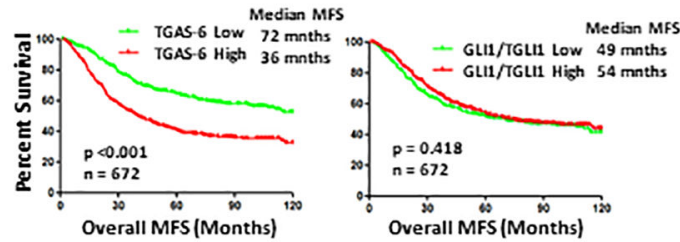

$N=\begin{array}{cccc}152 & 95 & 96 & 166\end{array}$
B

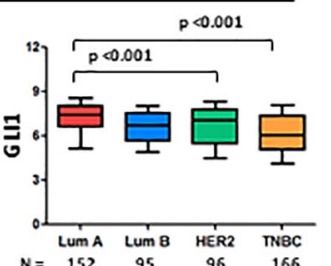

\section{D}
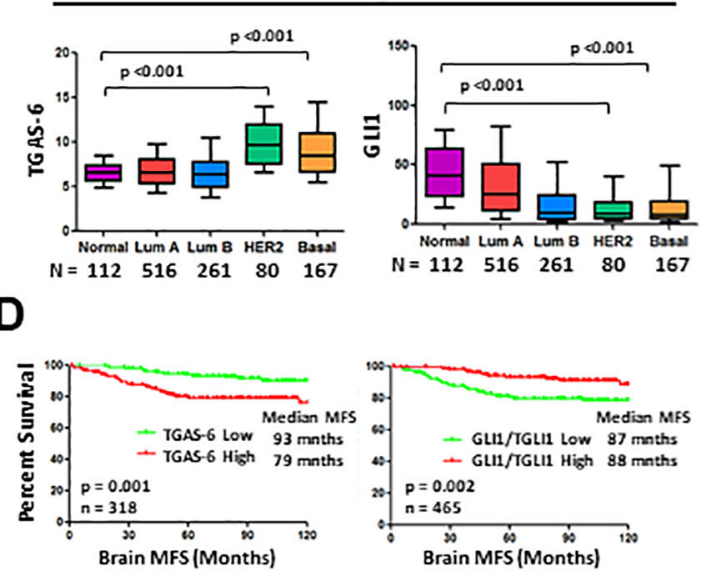

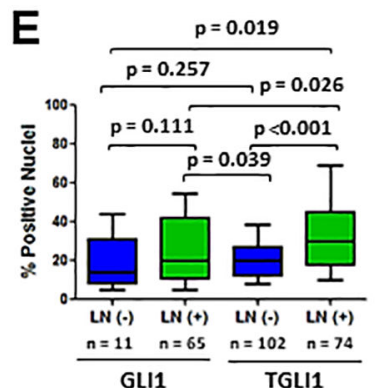

$\mathbf{F}$
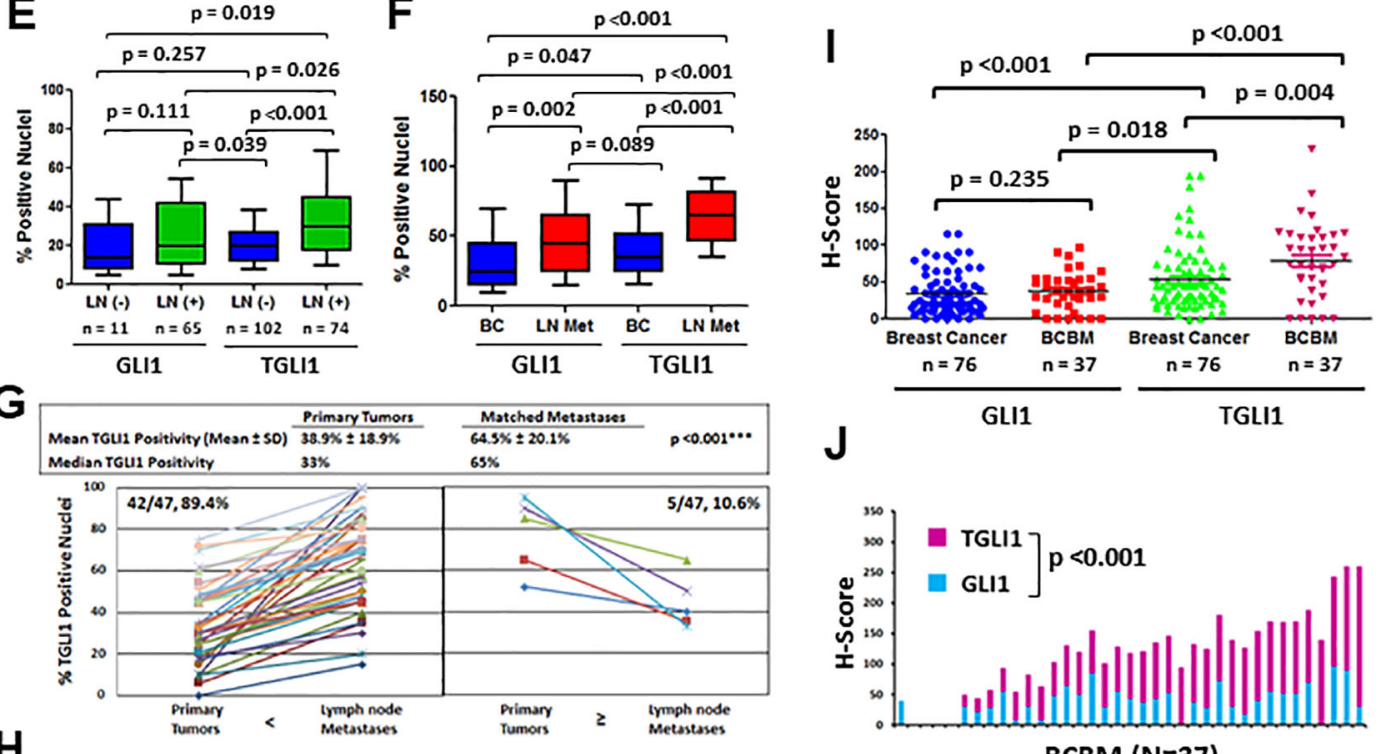

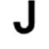

$\mathrm{H}$
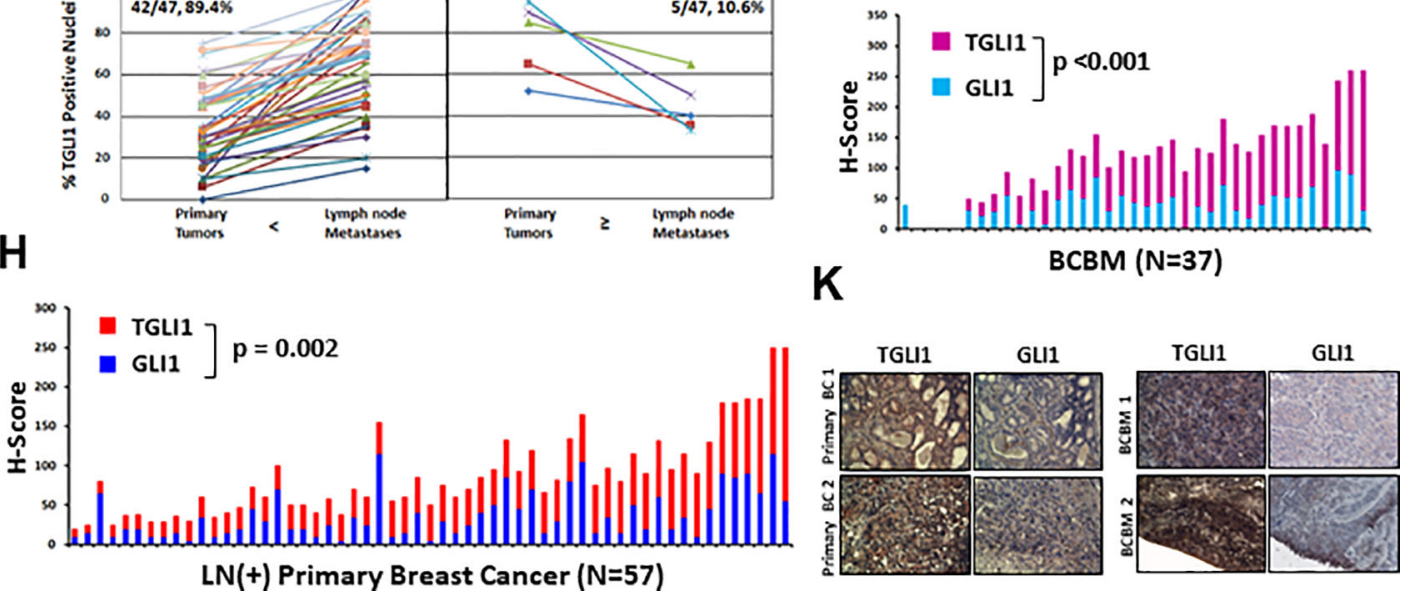

Figure 3: TGLI1 activation is enriched in HER2-enriched breast cancer and TNBC, and associated with poor MFS; TGLI1 expression is increased in lymph node metastases and BCBM specimens.

A-B) Bioinformatic analysis of GEO datasets (GSE12276/2034/2603/5327/14020; Panel A) comprised of 710 breast tumors and TCGA dataset (Panel B) comprised of 1,136 breast tumors for tGAS-6 enrichment and GLI1/TGLI1 mRNA expression. C-D) Kaplan-Meier curves showing the correlations between tGAS-6 or GLI1/TGLI1 mRNA expression with overall MFS (Panel C) and brain MFS (Panel D). Only 672 out of 710 patients in the GEO cohort had survival data. Log rank test was used to compute p-values. E-K) IHC analysis of 
patient specimens using TGLI1 and GLI1 specific antibodies developed in our laboratory. Panel $\mathbf{E}$ shows the expression of GLI1 and TGLI1 in primary breast tumors that are either lymph node-negative, LN(-), or lymph node-positive, LN(+). Panels F-G, expression levels of GLI1 and TGLI1 in matched primary breast cancers and lymph node metastases $(\mathrm{N}=47)$.

Panel $\mathbf{H}$ shows that TGLI1 expression is higher than GLI1 in LN(+) breast cancer in a pairwised analysis (N=57). Panel I shows GLI1 and TGLI1 expression in 56 primary breast tumors and 37 BCBM specimens. Panel J contains a pairwise analysis of 37 BCBM for GLI1 and TGLI1 expression; Panel K shows representative primary breast cancer and BCBM samples immunostained for GLI1 and TGLI1. Student's $t$-test was used to compute p-values. 
A

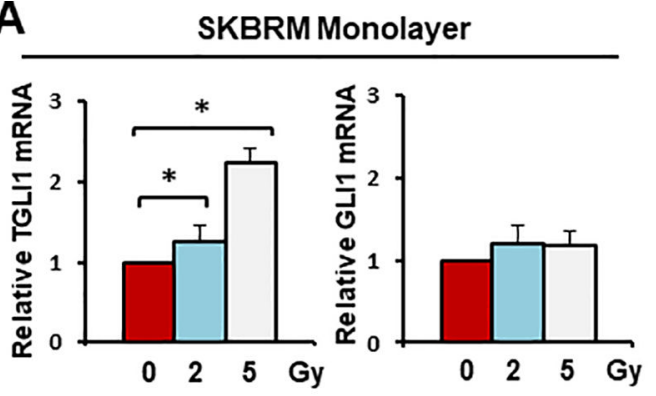

B

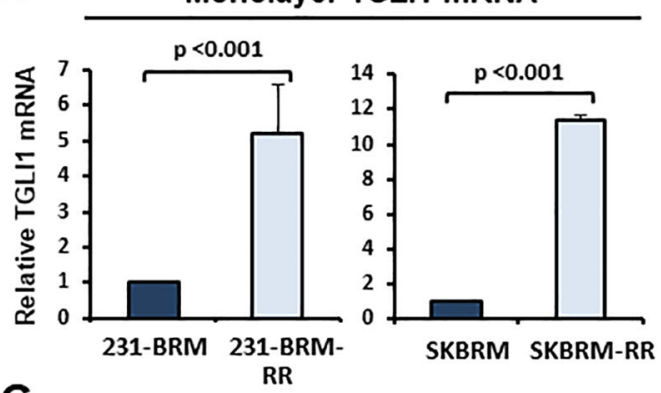

C
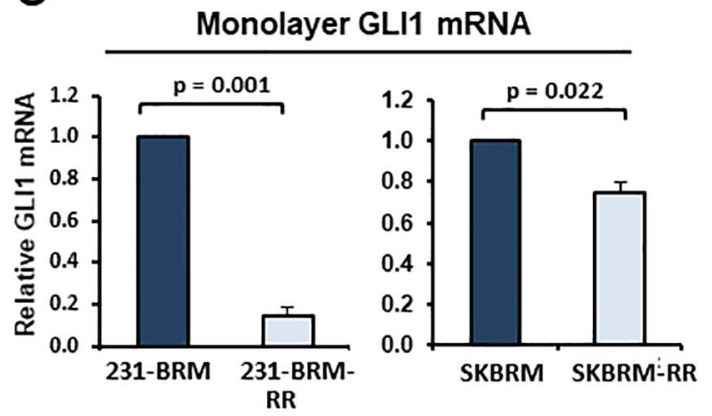

D
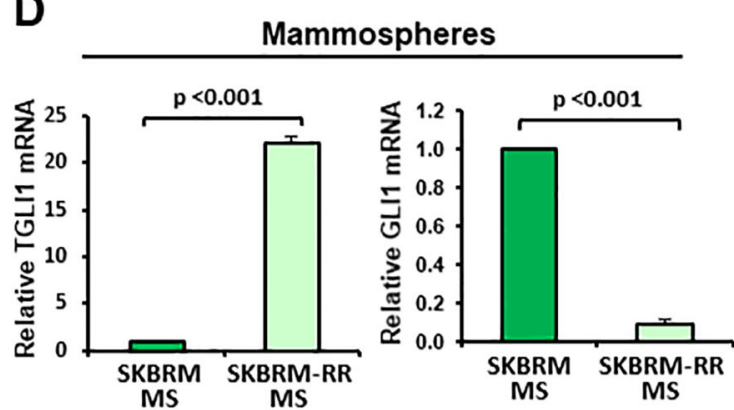
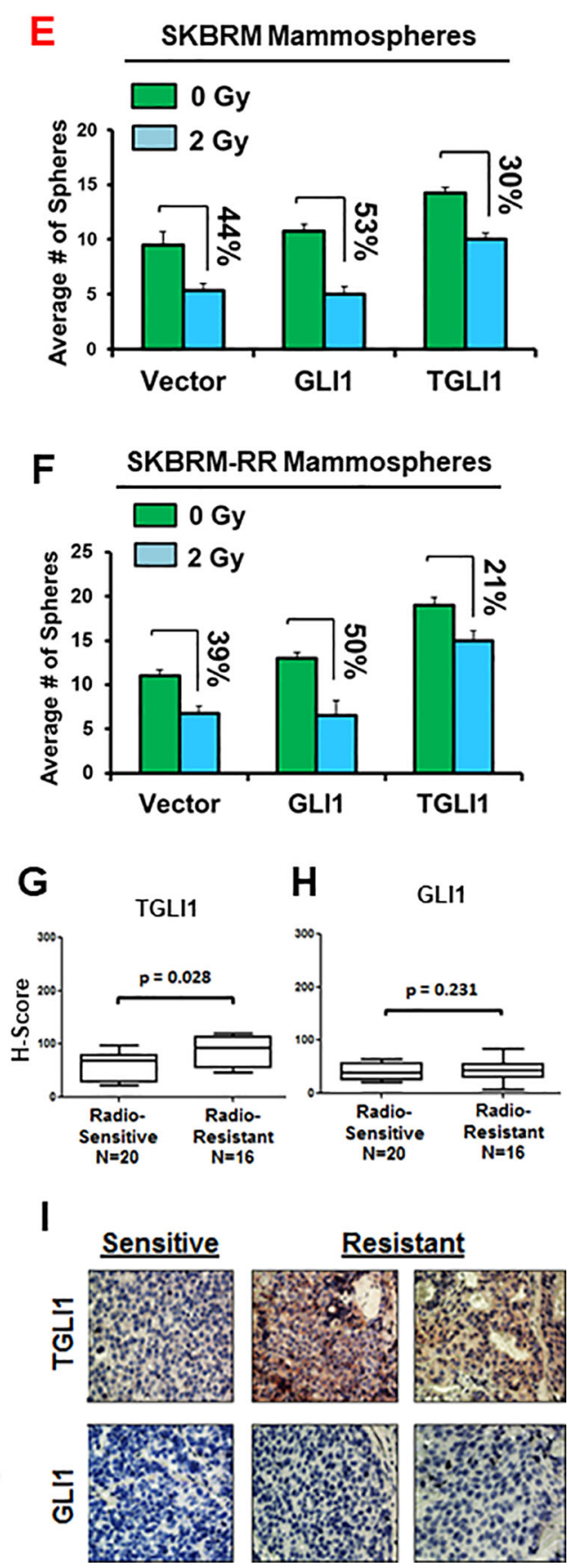

Figure 4: TGLI1 contributes to BCBM radioresistance.

A) Expression levels of TGLI1 and GLI1 in SKBRM cells treated with 0, 2, or 5 Gy of radiation. TGLI1 and GLI1 mRNA expression was determined 30 minutes after irradiation. B-C) Relative TGLI1 or GLI1 mRNA expression in radiosensitive and radioresistant braintropic breast cancer cell lines. D) TGLI1 mRNA was expressed at higher levels in SKBRMRR mammospheres than SKBRM mammospheres. GLI1 expression was significantly decreased in the radioresistant mammospheres. E-F) SKBRM and SKBRM-RR cells transfected with control, GLI1-, or TGLI1-vector were irradiated (2 Gy) and subjected to 
mammosphere formation assay. G-H) IHC staining of 20 radiosensitive BCBM tumors and 16 radioresistant BCBM tumors using GLI1 and TGLI1 specific antibodies developed in our laboratory. I) Representative immunostained BCBM specimens. *Indicates significant difference $(\mathrm{p}<0.05)$. Student's $t$-test was used to compute $\mathrm{p}$-values. 
F

J
A

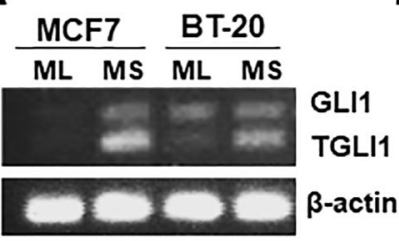

ML: Monolayer

MS: Mammosphere
D

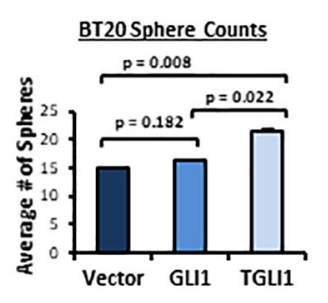

E

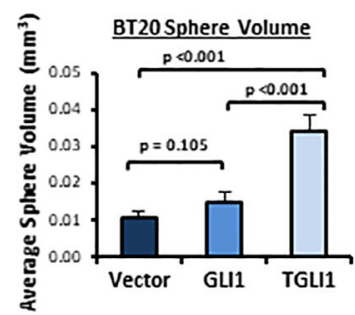

B
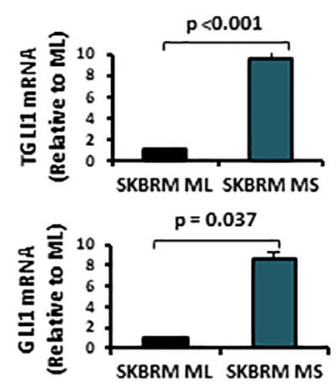

G
C

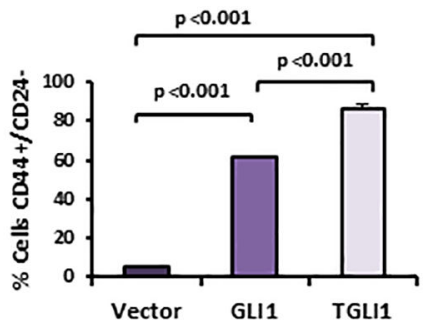

$\mathrm{H}$

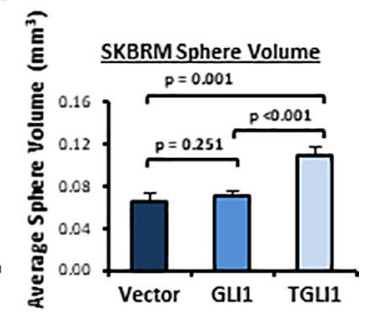

BT20 Mammospheres
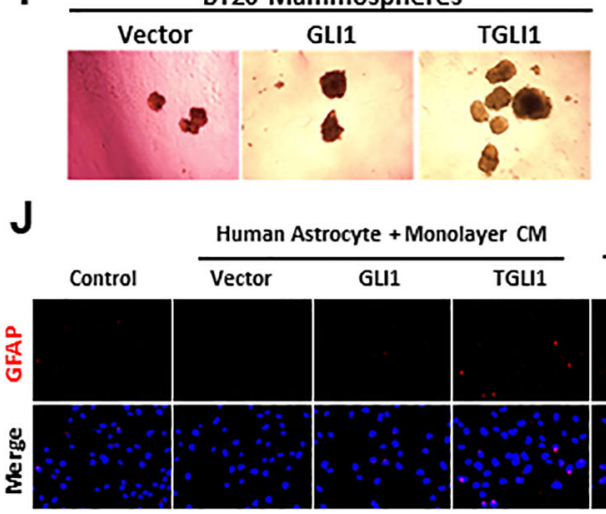

Human Astrocyte + Mammosphere CM

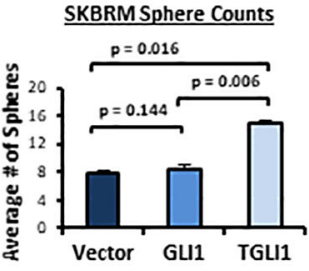

SKBRM Mammospheres

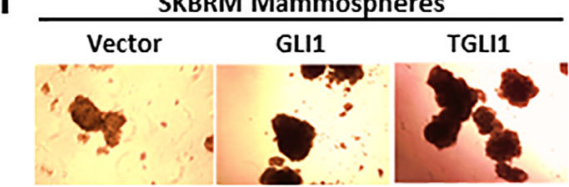

K

$\square \mathrm{ML} \square \mathrm{MS}$
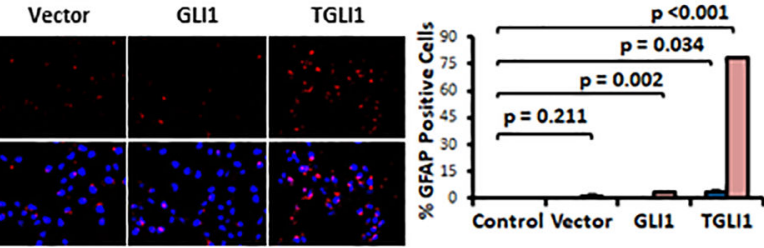

L Human

M
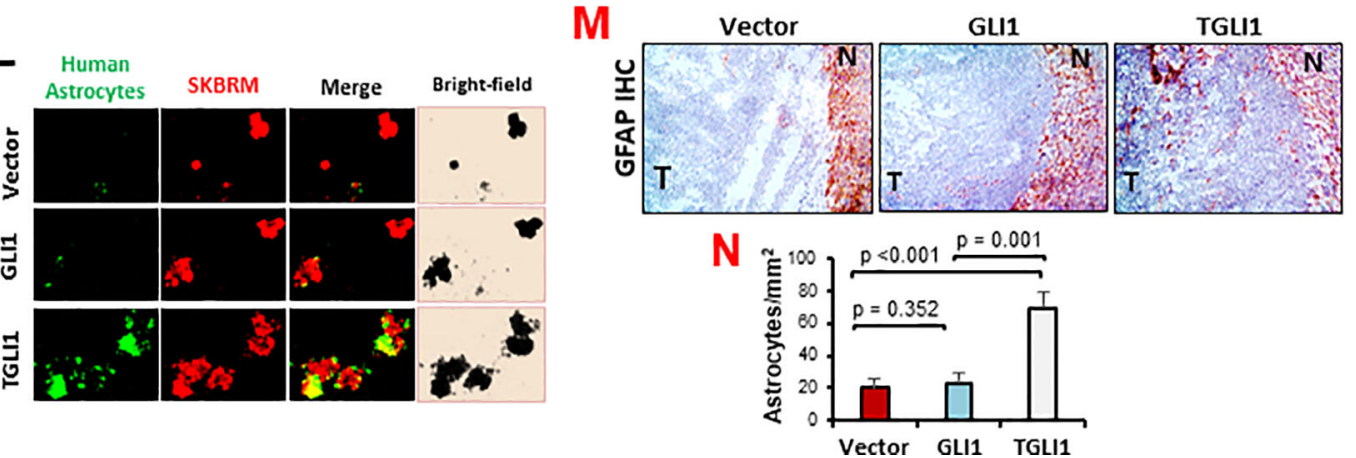

Figure 5: TGLI1 promotes breast cancer stem cells and TGLI1-positive stem cells activate human astrocytes.

A) Expression of GLI1 and TGLI1 mRNA in a two breast cancer cell lines grown in monolayer or mammospheres. Regular RT-PCR was conducted. MS, mammosphere. ML, monolayer. B) GLI1 and TGLI1 mRNA expression in SKBRM MS relative to ML, as determined by qRT-PCR. C) TGLI1 overexpression in MDA-MB-231 cells led to increased stem cell population defined by CD44 $4^{\text {high} / C D 24 l o w ~ a s ~ d e t e r m i n e d ~ b y ~ F A C S . ~ D-F) ~ T G L I 1 ~}$ ectopic expression enhanced the mammosphere-forming ability of BT20 cells. Panel D, 
average number of mammospheres. Panel E, average size of mammospheres. Panel F, representative images of mammospheres. G-I) TGLI1 ectopic expression enhanced the mammosphere-forming ability of SKBRM cells. Panel G, average number of mammospheres. Panel H, average size of mammospheres. Panel I, representative images of mammospheres. J-K) GFAP expression is increased in human astrocytes upon stimulation with conditioned medium (CM) from TGLI1-positive mammospheres. Isogenic SKBRM cells grown in monolayer or mammospheres were used. Panel $\mathrm{J}$, representative images. Panel K, percent GFAP positive cells. L) Co-culture of human astrocytes (GFP-tagged) with isogenic SKBRM cells (RFP-tagged) under the sphere-forming condition. M-N) Analysis of isogenic MDA-MB-231 intracranial tumors for activated astrocytes (GFAP IHC). M) Representative images. N) Quantified number of astrocytes per area of tumor. Student's $t$ test was used to determine $\mathrm{p}$-values. 
A
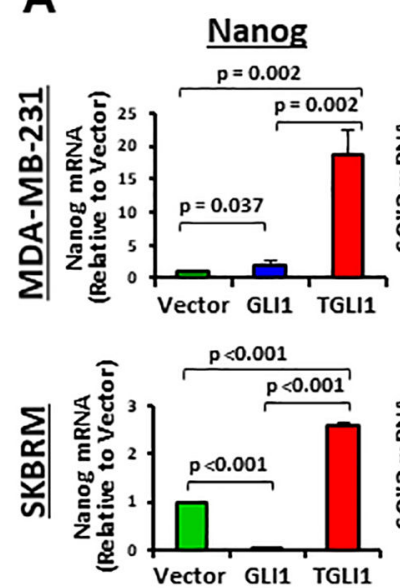
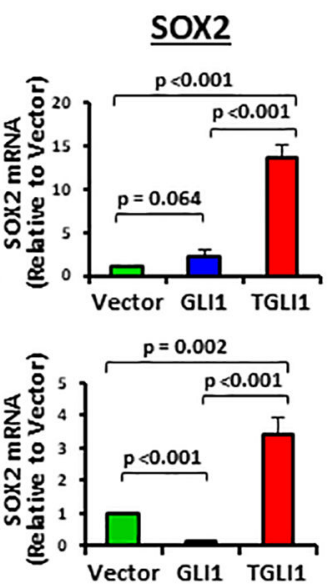

B

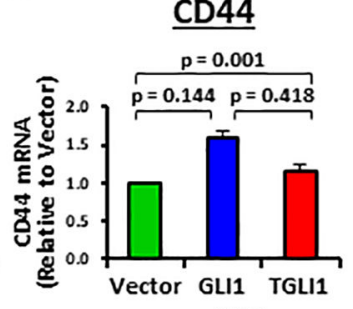

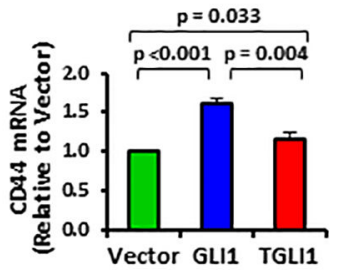

C
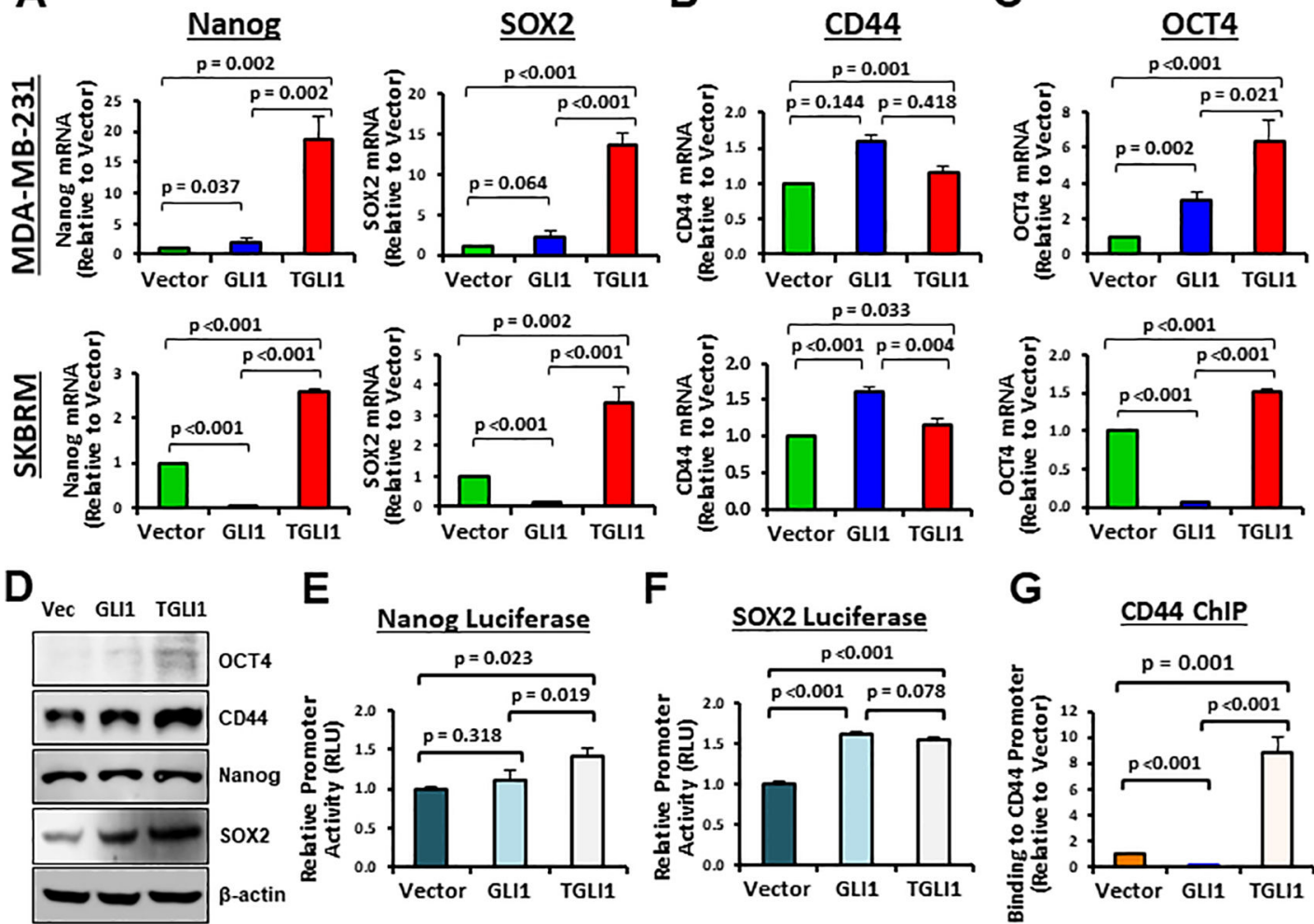

E

Nanog Luciferase

$F$
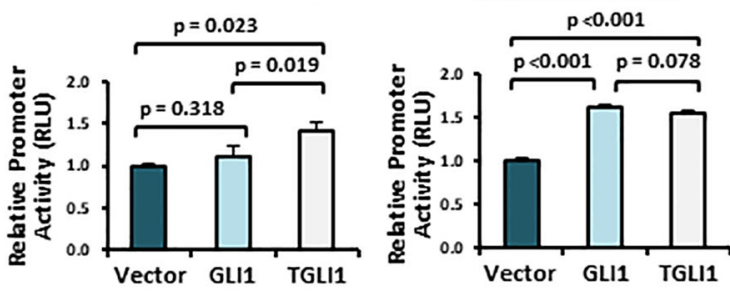

G
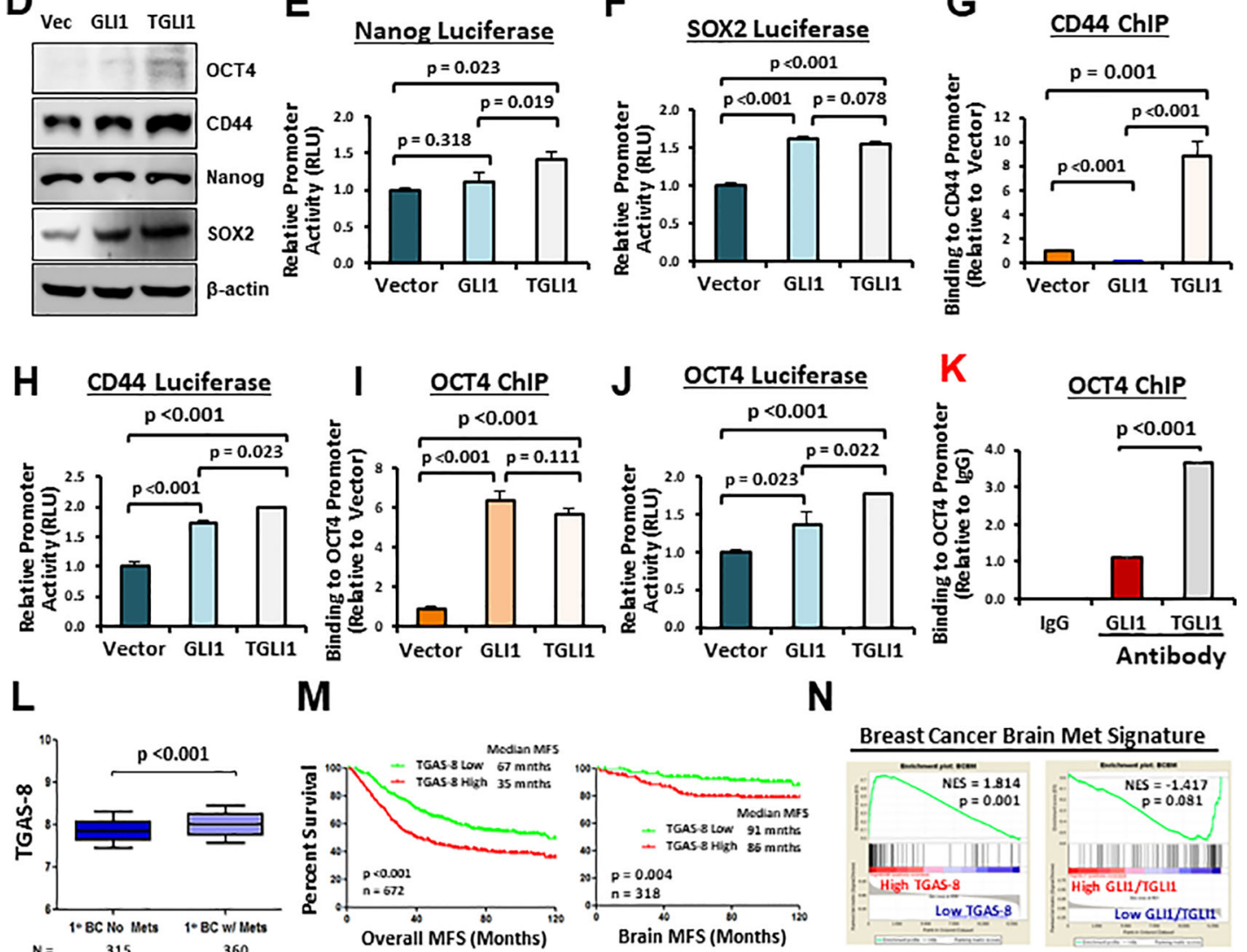

M

$\mathbf{N}$
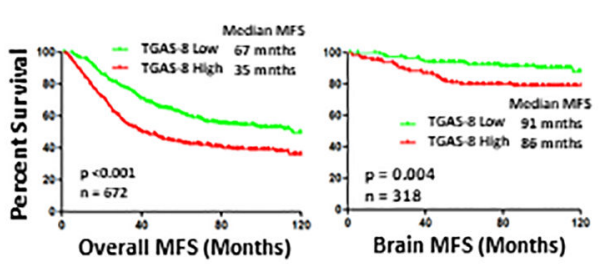

Breast Cancer Brain Met Signature

Figure 6: TGLI1 upregulates stemness genes in breast cancer cells; Eight-gene TGLI1 activation signature (tGAS-8) is predictive of breast cancer metastasis and radioresistance.

A-C) TGLI1 upregulates expression of four stemness genes in two breast cancer cell lines of different subtypes. Quantitative RT-PCR was performed using transfected MDA-MB-231and SKBRM cells. D) Protein levels of all four stemness genes were enhanced by TGLI1 in SKBRM cells. Western blotting was conducted. E\&F) SKBR3 cells were transiently transfected with promoter luciferase reporters and vector, GLI1 or TGLI1 plasmids, stimulated with SHH ligand $(100 \mathrm{ng} / \mathrm{mL})$ for $4 \mathrm{hrs}$ and promoter transactivation was 
measured by luciferase assay. E) Relative transactivation of the Nanog promoter by GLI1 or TGLI1. F) Relative transactivation of the SOX2 promoter by GLI1 or TGLI1. G) Relative binding of GLI1 or TGLI1 to the CD44 promoter as measured by the ChIP assay using transfected SKBR3 cells. H) Relative transactivation of the CD44 promoter by GLI1 or TGLI1 in SKBR3 cells. I) Relative binding of GLI1 or TGLI1 to the OCT4 promoter in SKBR3 cells as determined by the ChIP assay. qPCR was performed using primers spanning the OCT4 gene promoter. J) Relative transactivation of the OCT4 promoter by GLI1 or TGLI1 in SKBR3 cells as determined by the luciferase reporter assay. K) Relative binding of GLI1 or TGLI1 to the OCT4 promoter in MDA-MB-231 cells with dual overexpression of GLI1 and TGLI1 as determined by the ChIP assay using GLI1-specific and TGLI1specific antibodies developed and validated in our laboratory. Student's $t$-test was used to determine p-values. L-M) The same GEO cohort of 710 breast tumors from Figure 3 was used. L) Metastatic breast tumors had higher tGAS-8 than non-metastatic counterparts. M) Patients with high tGAS- 8 have a shortened overall MFS and brain MFS compared to those with low tGAS-8. N) GSEA that determined whether breast tumors with high tGAS-8 or high TGLI1/GLI1 mRNA were enriched for breast cancer brain metastasis signature. NES, normalized enrichment score. 
A
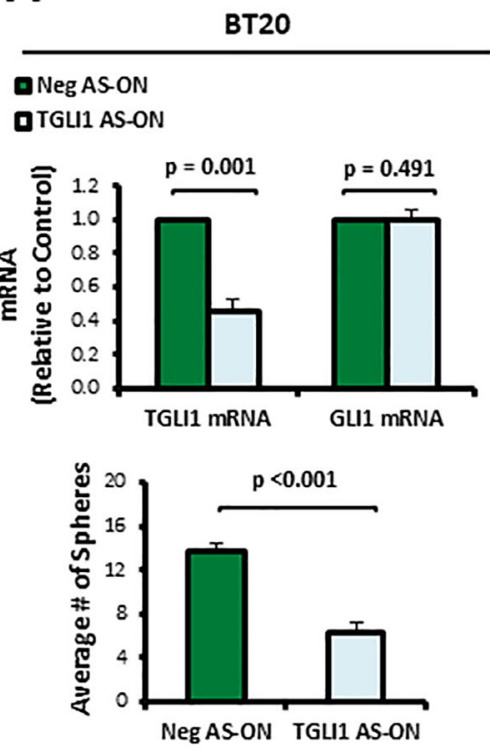

B

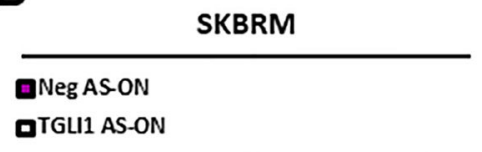

C
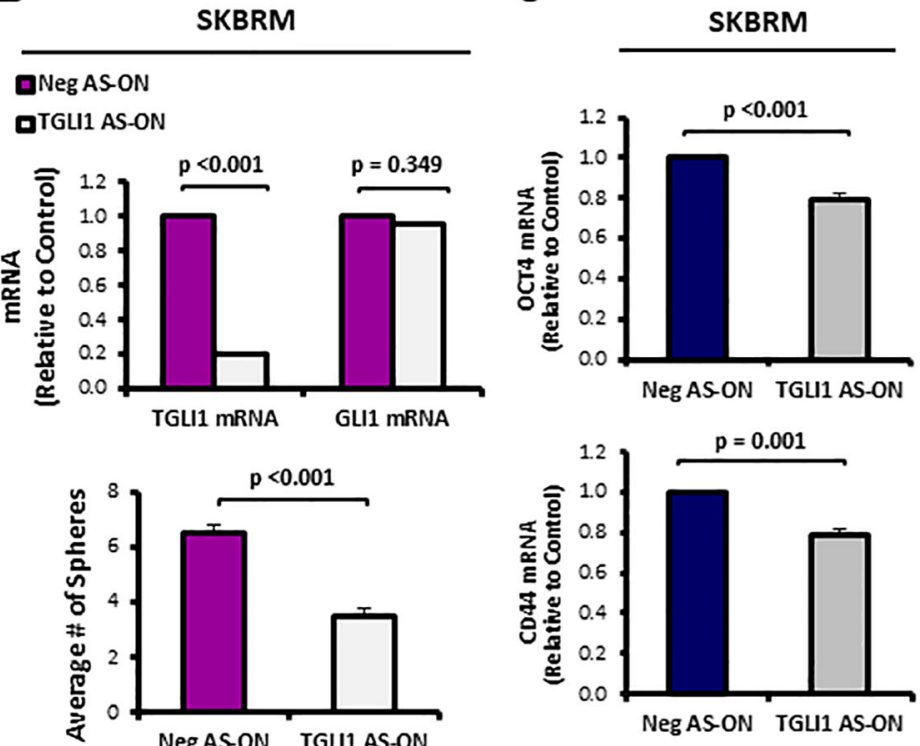

D

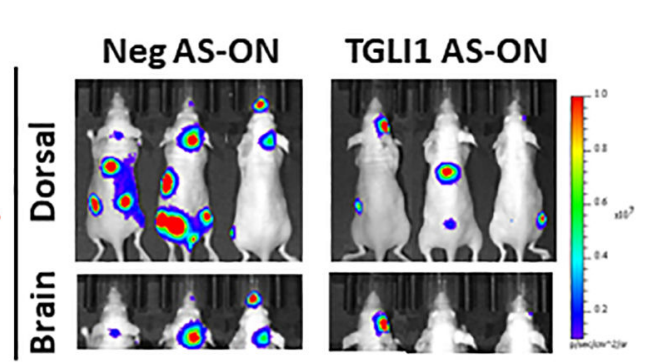

$\mathbf{F}$

Ex Vivo Brain Bioluminescence

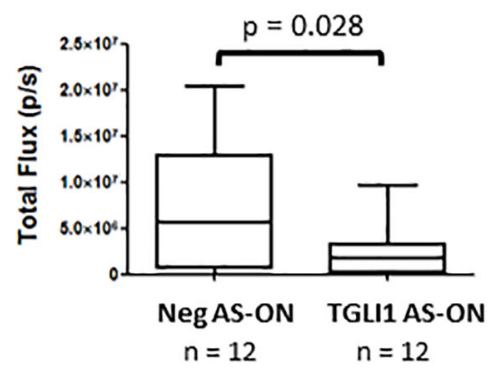

$\mathbf{E}$

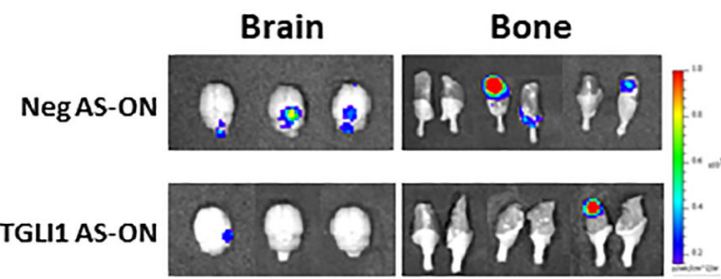

G

\section{Ex Vivo Bone Bioluminescence}

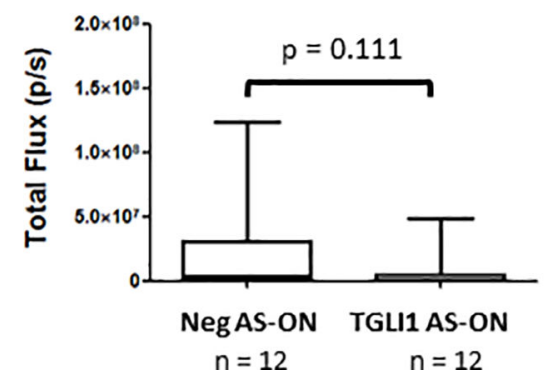

Figure 7: Knockdown of TGLI1 suppresses breast cancer stem cells in vitro and reduces BCBM in vivo.

Antisense oligonucleotides (AS-ON) were used to knockdown TGLI1. A-B) Selective knockdown of TGLI1 as indicated by qRT-PCR and effects of TGLI1 knockdown on mammosphere formation in A) BT-20 cells and B) SKBRM cells. C) Decreased expression of OCT4 and SOX2 with TGLI1 knockdown. Quantitative RT-PCR was performed using transfected SKBRM cells. In Panels D-G, athymic mice were inoculated with SKBRM cells transfected with negative control oligos or TGLI1-AS-ON through intracardiac injections 
(12 mice per group). D) Bioluminescent images of female athymic mice 25 days post intracardiac injection of transfected SKBRM cells. Dorsal and brain views are shown. E) Representative ex vivo IVIS images of brain and bone are shown. F) Brain ex vivo bioluminescence was quantified. G) Bone ex vivo bioluminescence was quantified. Student's $t$-test was used to compute p-values. 